BEACH EROSION BOARD OFFICE OF THE CHIEF OF ENGINEERS

\title{
A SUMMARY OF THE THEORY OF OSCILLATORY WAVES
}

TEGHNICAL REPORT NO. 2

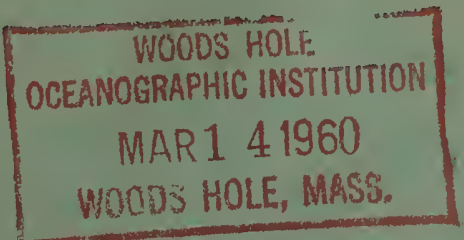

\section{$T C$ \\ 172}

.58

1942

\section{DOCUME:}

COLIEETHA 


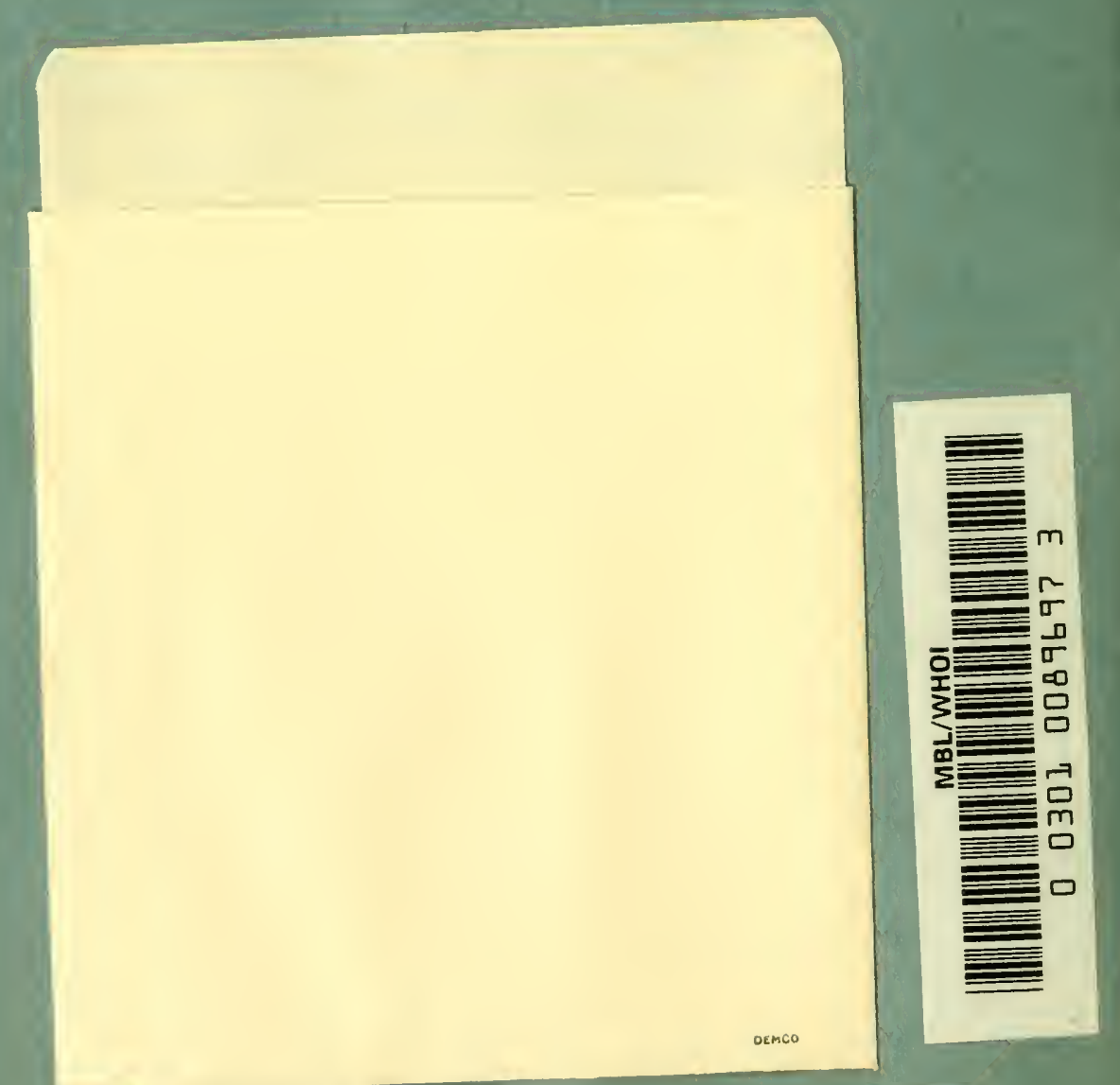




\section{A SUMMARY OF THE THEORY OF}

\section{OSCILLATORY WAVES}

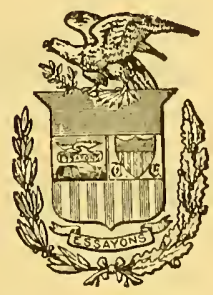

TECHNICAL REPORT NO. 2

BEACH EROSION BOARD

OFFICE OF THE GHIEF OF ENGINEERS 
WAR DEPARTMENT, Office of the Chief of Engineers,

Approved for publication.

November 21, 1941.

By authority of the Secretary of War:

E. REYBOLD,

Major General, Chief of Engineers.

(II) 


\section{WAR DEPARTMENT \\ OFFICE OF THE CHIEF OF ENGINEERS}

\section{BEACH EROSION BOARD}

The Beach Erosion Board of the War Department was established by section 2 of the River and Harbor Act approved July 3, 1930 (Public, 520, 71st Cong.), to cause investigations and studies to be made in cooperation with the appropriate agencies of various States on the Atlantic, Pacific, and Gulf coasts, and on the Great Lakes, and the Territories, with a view to devising effective means of preventing erosion of the shores of the coastal and lake waters by waves and currents. The duties of the Board were modified by an act approved June 26, 1936 (Public, 834, 74th Cong.), "to make investigations with a view to determining the most suitable methods of beach protection and restoration of beaches in different localities; to advise the States, counties, municipalities, or individuals of the appropriate locations for recreational facilities; and to publish from time to time such useful data and information concerning the protection of the beaches as the Board may deem to be of value to the people of the United States. * * * "

As of September 1940, the membership of the Beach Erosion Board is: Col. Jarvis J. Bain, senior member; Lt. Col. John F. Conklin; Lt. Col. Charles H. Cunningham; Maj. A. C. Lieber, jr., resident member; Prof. Thorndike Saville, Dean, College of Engineering, New York University; Gen. Richard K. Hale, Director, Division of Waterways, Department of Public Works, Boston, Mass.; and Prof. Morrough P. O'Brien, University of California. First Lt. William C. Hall is recorder of the Board. 


\section{TECHNICAL REPORTS}

Technical Report No. 1-A Study of Progressive Oscillatory Waves in Water, 1941.

Technical ReportNo. 2-A Summary of the Theory of Oscillatory Waves.

The authority for publication of this report was granted by an act for the improvement and protection of the beaches along the shores of the United States (Public, 834, 74th Cong.), approved June 26, 1936.

September 18, 1940.

This paper was prepared by Prof. Morrough P. O'Brien, member of the Board, with the assistance of Dr. Martin A. Mason and the staff of the Beach Erosion Board, in order to bring into one publication the various basic theories of oscillatory wave motion in water. While it was prepared primarily for departmental use, the Board believes that it will be of material value to engineering and research agencies engaged in work involving wave action.

A. C. Lreber, jr., Major, Corps of Engineers, Resident Member. 
List of symbols

List of references

1. Introduction

2. Characteristics of Oscillatory Waves........ 1

3. Waves of Small Amplitude...... 5

4. Waves of Finite Amplitude....... 14

A. Irrotational Theory .... 14

B. Trochoidal Theory ........ 24

5. Wave Groups and Transmission of Energy $\ldots \ldots \ldots$

6. Waves on a Sloping Bottom 35

7. Wave Refraction

8. Damping of Oscillatory Waves..... 41

\section{LIST OF SYMBOLS}

$Y=A$ vertical displacement of water particles.

$X=\mathrm{A}$ horizontal displacement of water particles.

$t=\mathrm{A}$ specific time.

$L=$ Wave length.

$C=$ Wave velocity.

$T=$ Wave period.

$g=$ Acceleration due to gravity.

$d=$ Water depth.

$\tau=$ Surface tension.

$\rho=$ Density of water.

$z=$ Depth below still water level.

$x=$ Displacement in direction of wave travel.

$y=$ Surface ordinate.

$h=$ Wave height from trough to crest.

$a_{z}, b_{z}=$ Horizontal and vertical displacements of a water particle

$E_{p}=$ Potential energy of wave per unit width of crest.

$E_{k}=$ Kinetic energy of wave per unit width of crest.

$w=$ Weight of water per cubic foot.

$a=$ Half of wave height.

$\bar{U}=$ Velocity of mass transport.

$\omega=$ Vorticity.

$R=$ Radius of rolling circle (Gerstner theory).

$r=$ Radius of tracing circle (Gerstner theory), or polar coordinate.

$\theta=$ Angular displacement (Gerstner theory), or polar coordinate.

$P=$ Power transmitted by wave.

$C_{Z}=$ Group velocity.

$\mu=$ Absolute viscosity of water.

$\nu=$ Kinematic viscosity of water.

$\phi=$ Potential function.

$\alpha=$ A function of wave height, or an angle.

$m=2 \pi / L$.

$n=$ An integral number.

$U=$ Horizontal component of velocity of water particle.

$W=$ Vertical component of velocity of water particle.

$G=$ Volume of mass transport per unit width of crest. 


\section{LIST OF REFERENCES}

1. Rayleigh, Lord-On Periodic Irrotational Waves at the Surface of Deep Water-Philosophical Magazine, Vol. XXXIII, 1917, pp. 381-389.

2. Stokes, G. G.-On the Theory of Oscillatory Waves-Transactions, Cambridge Philosophical Society, Vol. VIII, 1847, p. 441, and Supplement, Scient. Papers, Vol. I, p. 314.

3. Lamb, Horace-Hydrodynamics, 6th Ed., 1932, p. 367.

4. Lamb, Horace-Hydrodynamics, 6th Ed., 1932, p. 459.

5. Lamb, Horace-Hydrodynamics, 6th Ed., 1932, p. 368.

6. Lamb, Horace-Hydrodynamics, 6th Ed., 1932, p. 260.

7. Levi-Civita, T.-Détermination rigoureuse des ondes d'ampleur finieMathematische Annalen, Vol. XCIII, 1925, pp. 264-314.

8. Struik, D. J.-Détermination rigoureuse des ondes irrotationelles périodiques dans un canal à profondeur finie, Mathametische Annalen, Vol. XCV, 1926, pp. 595-634.

9. Lamb, Horace-Hydrodynamics, 6th Ed., 1932, p. 419.

10. Mitchim, C. F.-Oscillatory Waves in Deep Water, The Military Engineer, March-April, 1940.

11. Michell, J. H.-On the Highest Waves in Water, Philosophical Magazine, (5), Vol. XXXVI, 1893, pp. 430-437.

12. Havelock, E. T.-Periodic Irrotational Waves of Finite Height-Proceedings of the Royal Society, London, Series A, Vol. 95, 1918, pp. 38-51.

13. Rayleigh, Lord-Hydrodynamical Notes-Philosophical Magazine, Vol. XXI, 1911 , pp. 177-195.

14. Gerstner, Franz-Theorie der Wellen-Abhandlungen der königlichen böhmischen Gesellschaft der Wissenschaften, Prague, 1802; also Gilbert's Annalen der Physik, Vol. XXXII, 1809, pp. 412-445.

15. Rankine, W. J. M.-On the Exact Form of Waves Near the Surface of Deep Water-Philosophical Transactions of the Royal Society, London, 1863, p. 127.

16. Gaillard, D. D.-Wave Action in Relation to Engineering Structures-1904, reprinted at Engineer School, Fort Belvoir, Va., 1935.

17. Lamb, Horace-Hydrodynamics, 6th Ed., 1932, p. 381.

18. Lamb, Horace-Hydrodynamics, 6th Ed., 1932, p. 237.

19. Reynolds, Osborne-On the Rate of Progression of Groups of Waves and the Rate at which Energy is Transmitted by Waves-Nature, Vol. XVI, 1877, pp. 343-344; also Scientific Papers, Vol. 1, p. 198.

20. Rayleigh, Lord-On Progressive Waves-Proceedings London Mathematical Society, Vol. IX, 1877, pp. 21-26; also Scientific Papers, pp. 322-327.

21. Lamb, Horace-Hydrodynamics, 6th Ed., 1932, p. 382.

22. Wien, W.--Lehrbuch der Hydrodynamik, Leipzig, 1900, Section 3, pp. 201212.

23. Rayleigh, Lord-Hydrodynamical Notes-Philosophical Magazine, Vol. XXI, 1911, pp. 177-195.

24. Airy, G. B.-On Tides and Waves-Encyclopaedia Metropolitana, London, 1842, p. 289.

25. Ivanov, A. A.-Bulletin, Academy of Sciences, U. S. S. R., Sir. Geogr. Nr. 5/6, pp. 477-490.

26. Lamb, Horace-Hydrodynamics, 6th Ed., 1932, p. 623.

27. Hough, S. S.-On the Influence of Viscosity on Waves and Currents-Proceedings, London Mathematical Society (1), Vol. XXVIII, 1896, pp. 264288. 


\section{A SUMMARY OF THE THEORY OF OSCILLATORY WAVES}

\section{Section 1. INTRODUCTION}

Quantitative predictions of the effect of wave action in shore-line processes requires, first, statistical information on the wave height, length, period, and direction, and water depth, at each locality for each seeson; and second, a precise knowledge of the behavior of waves approaching a shore line through deep water, into and through shoaling water. This paper deals with the second phase only, presenting a summary of the theoretical treatments of oscillatory wave motion on a free water surface. It is limited to the type of wave encountered in shore protection problems. Much of the theory requires experimental verification, and mention will be made of the extent to which confirmation has been obtained, without, however, quoting experimental results.

The derivations of equations, where not given herein, are to be found in the references quoted.

\section{Section 2. CHARACTERISTICS OF OSCILLATORY WAVES}

All wave phenomena have some elements in common and it is important to establish at the outset the approximate boundaries of the subject to be covered by this summary. The term oscillatory has been used to emphasize the periodic character of the phenomenon and to eliminate the ideas associated with the term "wave of translation." The waves under consideration induce a steady state of oscillation which differs in many particulars from the transient state characteristic of a wave of translation. Tides and seiches are also periodic but are of such length in comparison with the water depth as to permit simplifications in analysis which are not valid for the shorter period surface waves to be considered herein. Ripples are periodic and are produced by the wind but are of no consequence in shore-protection problems representing an initial phase in the generation of wind waves. Solitary and seismic waves present an essentially different problem and are not considered here. The waves under consideration are periodic disturbances under the control of gravity and inertia and of such height and period as to break on a sloping shore line.

To be more specific, the waves to be discussed are those which break on the shore line, throw sand into suspension, and possibly generate littoral currents. Periods are usually of the' order of 4 to 12 seconds along ocean shore lines but may be much greater. Heights 
vary widely with wind action, period, and bottom conditions, ranging from a few inches to many feet. Wave crests in deep water may be inclined at an angle with the shore line, parallel to the shore at the plunge point. Several wave trains of different periods and heights may simultaneously approach the shore from different directions, giving rise to a beat frequency in the breakers-this is the basis for the common observation that waves "break in sets." Under some conditions the wave energy may not be completely dissipated in the breakers and the plunge and an appreciable portion may return seaward in the form of a reflected wave. In all cases the observed wavo pattern may be considered as the result of superposition of primary and reflected waves of different wave trains, each periodic and of approximately constant amplitude. Accordingly, the first step in the analysis of oscillatory waves in water is to study the behavior of single wave trains of uniform period and amplitude as they progress over horizontal or sloping bottoms.

Although the motion in a breaking or plunging wave is too complicated for present methods of analysis, the essential periodic character of the waves is retained and the motion is oscillatory, in the broad sense of the term, from deep water to the limit of uprush. It should be noted that the plunge of the wave is the final break before the uprush on the beach.

The creation of oscillatory waves is probably the result of wind traction on the water surface. Once disturbed, pressure differences develop on the leeward and windward sides of the crest and permit a direct transfer of energy from the wind to the water through the medium of normal as well as tangential stresses. The details of the mechanism by which a small wave builds up into a large one should be investigated experimentally, but for the present it is sufficient to note that energy is continually transferred from the atmosphere to the surface layers of the ocean and that the waves thus formed provide the mechanism which transfers this energy to the shore line. From theory and experiment it is known that ocean waves in deep water travel great distances without appreciable loss of energy by internal friction. It is evident that on the average the waves observed at any point along the shore will be more controlled by distant than local winds.

The form of oscillatory waves depends upon the ratio of height to length and upon the local wind effect. Very long, low swells from distant storms are almost, but not exactly, sinusoidal in deep water. As they are telescoped by the retarding effect of a shoaling bottom, the crests become sharper while the troughs flatten and the form is then approximately that of a trochoid. Short waves under the influence of local wind are usually assymetrical or may be trochoidal. 
At the plunge point the combination of height, bottom slope, depth, and period reaches a limit for a stable wave form and the crest plunges.

Considering the progress of a single wave train of uniform height and length from deep water to the shore over a gradually sloping bottom, it seems reasonable to assume that the same period would be observed at all points along the path. This statement is by no means axiomatic, for waves often coalesce or break up into shorter waves; however, laboratory experiments on small waves moving over limited reaches show that the individual wave crests maintain their identity. The point raised is important in the application of wave equations to field conditions.

From the theoretical standpoint Airy concluded that the conditions of constant pressure and continuity could not be satisfied simultaneously on a sloping bottom, and from experiment it is known that an abrupt change in depth may cause waves to break up into shorter waves. In groups including a finite number of waves, as in the wave pattern around a ship, it is observed that waves at the front of the train disappear while new waves develop at the rear. A frequently observed similar phenomenon, where a wind wave can be followed for a considerable distance and then seems to disappear, probably results from the superposition of wave trains of different periods. The fundamental question is whether the component waves of each wave train maintain their identity. If they do, it follows that their period is the same at every point along the path even though the apparent period of the combined wave trains may vary from point to point.

The question of permanence of oscillatory waves has been the subject of much speculation and it is of interest to review some of the conclusions drawn. Rayleigh (1) stated that:

* * * I think that the reader who follows the results of the calculations here put forward is likely to be convinced that permanent waves of moderate height do exist.

It may be mentioned that most of the authorities **** express belief in the existence of permanent waves, even though the water be not deep, provided of course that the bottom be flat.

Stokes came to a similar conclusion as follows:

In fact it will presently appear that it is only an indefinite series of waves which possesses the property of being propagated with uniform velocity and without change of form; at least this is the case when the waves are such as can be propagated along the surface of a fluid which was previously at rest.

It appears from the above, that of all waves for which the motion is in two dimensions, which are propagated in a fluid of uniform depth, and which are such as could be propagated in a fluid previously at rest, there is only one particular kind, namely, that just considered, which possesses the property of being propagated with a constant velocity and without change of form; so that a solitary wave cannot be propagated in this manner.

$7796400-48-2$ 
The bottom slopes encountered off sandy coasts are usually gradual and, when plotted to true scale, seem almost horizontal. Whether or not the theories applicable to a horizontal bottom may be applied to these very gradual slopes remains to be determined by experiment or direct observations. Though identity of individual wave crests seems to be maintained over a gradually sloping bottom even this feature should be investigated.

The relationship between period, length, and velocity characteristic of all wave phenomena is

$$
L=C T
$$

This formula is essentially a definition. Independent measurements of length, velocity, and period should agree with equation 1 exactly; and deviations therefrom should be considered as observational errors. Agreement with this equation, however, is not a confirmation of the dynamical theories of oscillatory waves.

Oscillatory waves on the surface of water are characterized by periodic variations in the motion of the water particles, pressure, and surface elevation, each quantity passing through a series of values and returning to its initial value at regular intervals of time. At intervals equal to the wave length maasured in the direction of motion, each of these quantities will be equal to and in phase with its former value. From the standpoint of the mathematical analysis, the approximately constant atmospheric pressure on the water surface and the existence of appreciable vertical accelerations are of basic importance. The amplitude of orbital motion decreases exponentially below the surface and becomes very small at a depth equal to half the wave length and for most purposes the orbital velocity is inappreciable at even smaller ratios of depth to length. The effect of decreasing depth is a continuous function. Although some authors give a contrary impression, there is therefore no sharp line of demarcation between shallow-water and deep-water waves. The dividing point is purely arbitrary and is dependent upon the desired accuracy of computation. No abrupt change occurs when the depth is equal to half the wave length and the present arbitrary division of deepwater and shallow-water waves on the basis of the depth-length ratio is meaningless.

Present-day wave theory deals with waves of stable form in which all elements of the wave profile advance with the same velocity relative to the undisturbed water. From a theoretical standpoint, the wave motion is unchanged if the water is imagined to move relative to a fixed point at a velocity equal and opposite to the wave velocity, thus bringing the waves to rest relative to the same point of observation. The analysis is thus changed to one of steady flow in which the surface form, and distribution of horizontal and vertical accelerations must be so distributed as to satisfy the equation of 
continuity, and the condition of constant surface pressure. In addition, if the water is assumed to be frictionless, which it very nearly is for purposes of wave theory, the flow must be irrotational. The problem is thus reduced to the determination of an analytical expression, or the construction of a flow net, to satisfy these steady conditions. Continuity requires that the flow system in each small element of the wave be so constructed as to indicate no destruction or creation of material. The condition of constant surface pressure is very nearly satisfied for waves moving without wind action, and surface pressure differences due to wind are probably not great as compared with the hydrostatic pressure differences. Waves lose energy so slowly that the assumption of constant energy along each streamline seems reasonable. Irrotational motion occurs in fluids devoid of viscosity and this condition may not be entirely satisfied in natural waves; if the motion is not irrotational, the energy may be different along different streamlines. For a complete discussion of rotational and irrotational motion the reader is referred to Lamb, "Hydrodynamics," or Prandtl-Tietjens, "Fundamentals of Hydro- and Aero-Mechanics."

The aim of the classical analysis of wave motion has been to find the surface form and wave velocity which satisfy the conditions outlined.

\section{Section 3. WAVES OF SMALL AMPLITUDE}

The observed permanence of oscillatory waves is utilized for the mathematical analysis of the oscillatory wave problem. The solution then requires the determination of the dynamic conditions required for such permanence of wave form. This concept is fundamental to the analysis of wave motion of the type under consideration and its importance accounts for the preceding discussion of whetler or not waves actually do maintain their identity. Quantitatively, permannence of form requires that every element of the wave form advance with the same velocity. Consequently, a wave may be imagined as being brought to rest relative to a fixed reference system by superimposing a mass velocity in the water equal but opposite to the wave velocity. This does not modify the internal flow problem but does simplify the analysis to one of steady flow.

If water of constant depth is disturbed by small periodic impulses, the wave length and velocity are related to the depth by the equation (3).

$$
C=\sqrt{\frac{g L}{2 \pi} \tanh \frac{2 \pi d}{L}}
$$

So long as the surface curvature is everywhere large enough to eliminate the influence of surface tension and the wave height is smail as compared with $d$ and $L$, this equation applies to both deep-water and 
shallow-water waves. Combining equation 2 with equation 1 gives $C$ as a function of $d$ and $T$ (figure 1 ) or $L$ as a function of $d$ and $T$ (figures 2 and $2 \mathrm{a}$ ).

\section{RELATIONSHIP OF WAVE VELOCITY, PERIOD, AND DEPTH}

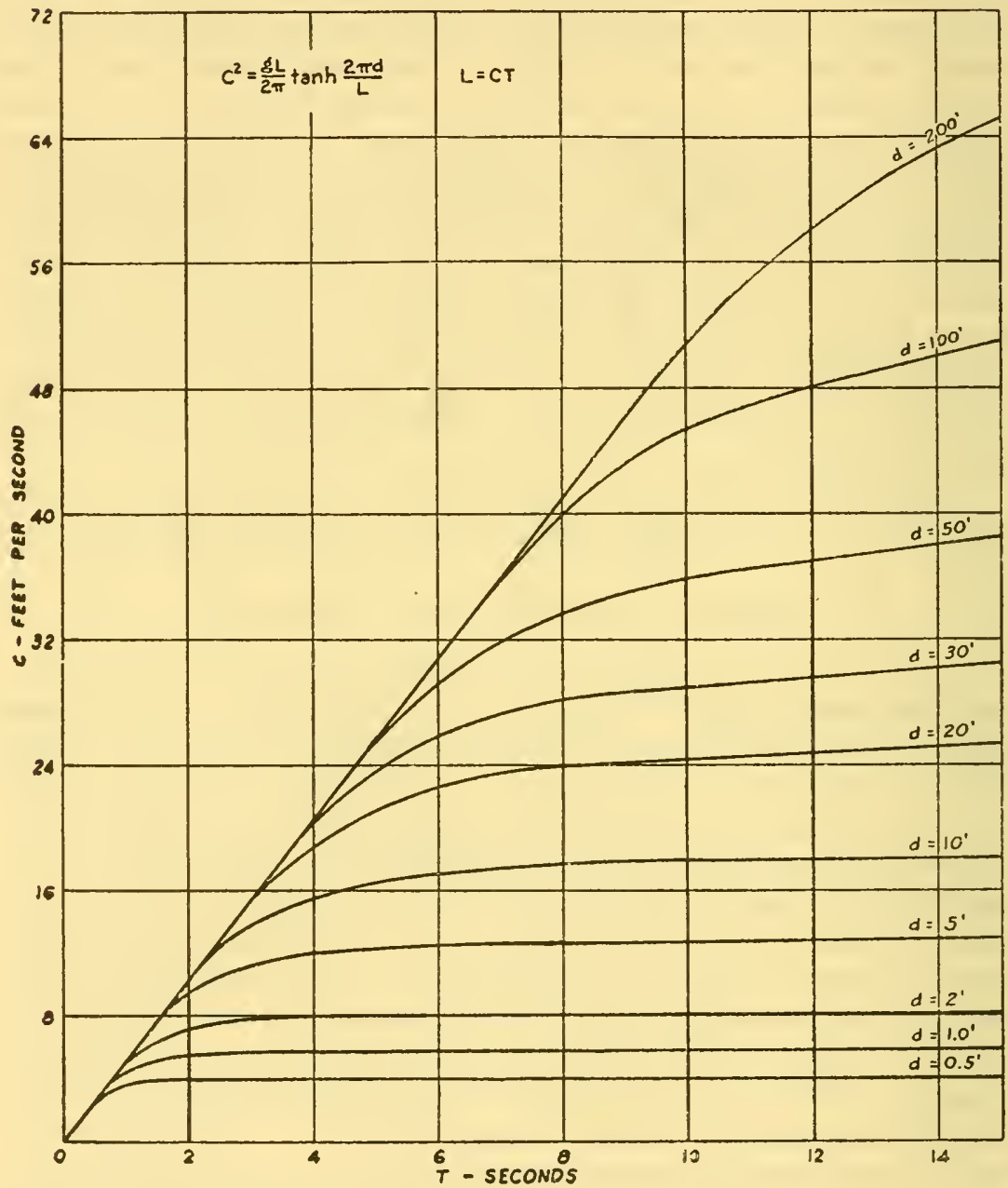

Fradre 1.

The effect of surface tension is negligible in nature but in model work it may be important, especially near the plunge point where the radius of surface curvature becomes very small. Experimental data will be necessary to establish the lower limit of size at which waves break in a manner similar to natural waves; however, some 
indication of the wave size for which surface tension is important is given by the equation for ripples (surface tension waves) (4).

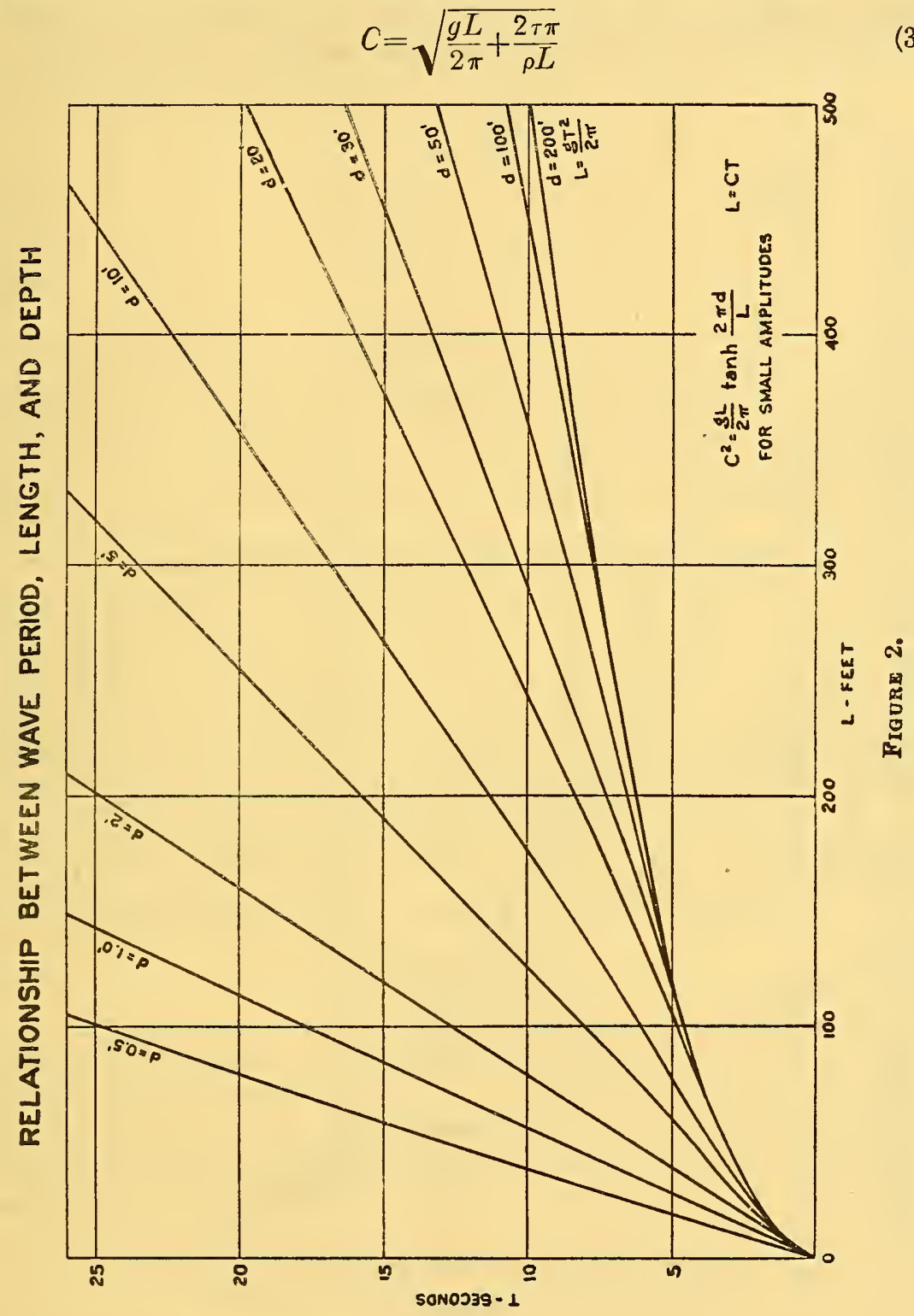

This equation shows a minimum point at

$$
C_{m i n}=\sqrt{2 \pi \frac{\tau}{\rho g}}
$$

or

$$
L_{m i n}=1.414 \sqrt[4]{\frac{\tau g}{\rho}}
$$


For water in contact with air at usual atmospheric temperatures, $\tau=0.0051$ pounds per foot; $L_{m i n}=0.056 \mathrm{ft}$; $C_{m i n}=0.756 \mathrm{ft} . / \mathrm{sec}$. When $L=3 L_{m i n}$, the wave velocity given by equation 3 differs from that given by equation 2 by less than 5 percent. The equations

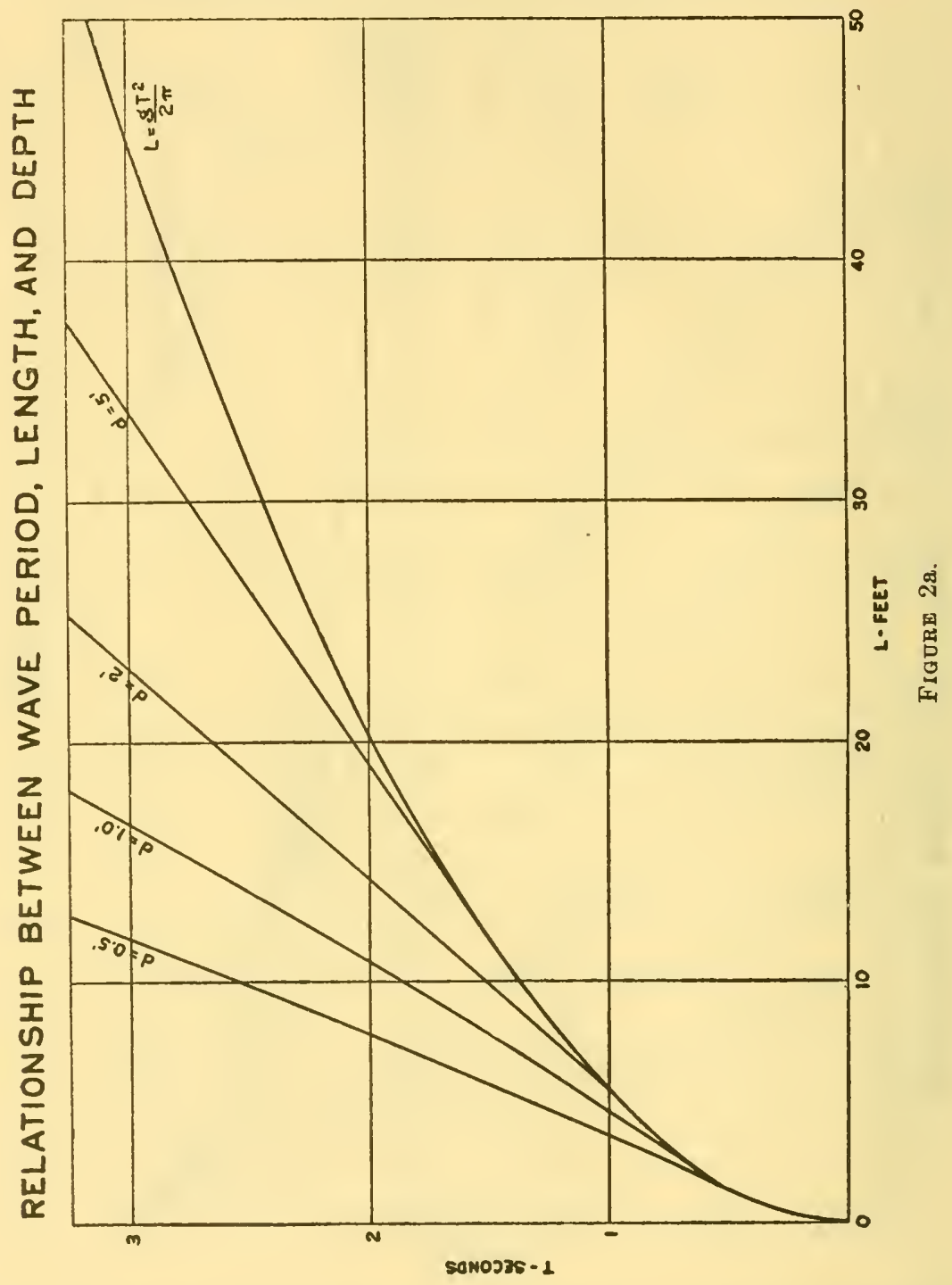

apply only to ripples of very small amplitude and no quantitative conclusions may be drawn from them regarding the effect of surface tension near the upper limit of the height-length ratio. Since a wave of 1-second period in deep water has a length of 5.1 feet, it is seen that the radius of curvature is so large in comparison to that 
of a ripple that the effect of surface tension is probably small even for waves of usual laboratory dimensions.

As $d$ becomes very large in equation 2 the term $\tanh \frac{2 \pi d}{L}$ approaches unity and the velocity expression becomes

$$
C=\sqrt{\frac{g L}{2 \pi}}
$$

since

$$
L=C T
$$

then:

$$
C=\frac{g T}{2 \pi}=5.12 T
$$

and

$$
L=5.12 T^{2}
$$

Equations $2 b$ and $2 c$ are the limiting curves in figures 1,2 , and $2 a$. As an example of the depth at which the bottom effect becomes negligible, let us assume $d=0.5 \mathrm{~L}$. Then tanh $(2 \pi \times 0.5)=0.9963$ and the wave velocity is 0.9963 times the velocity in an unlimited depth. If an error of 5 percent is permissible, the corresponding $\frac{d}{L}$ is 0.25 . Evidently, the limits of applicability of the deep-water wave equations depend on the accuracy desired and $\frac{d}{L}=0.5$ is a purely arbitrary point of division, though frequently mentioned in papers on wave phenomena as the basis of the definition that waves in water having a depth greater than half the wave length are deep-water waves, and waves in lesser depths are shallow-water waves.

If the wave length is very large as compared with the depth, $\tanh \frac{2 \pi d}{L}$ approaches $\frac{2 \pi d}{L}$ and equation 3 takes the form:

$$
C=\sqrt{\frac{g L}{2 \pi} \frac{2 \pi d}{L}}=\sqrt{g d}
$$

This is the well-known equation for the velocity of long waves of small amplitude in still water.

The horizontal and vertical displacements (3) of the water particles from their mean position at a distance $z$ below the still water level are functions of $x$ and $t$ as shown by:

and

$$
\begin{aligned}
& X_{z}=\frac{h}{2} \cdot \frac{\cosh \frac{2 \pi}{L}(d+z)}{\sinh \frac{2 \pi d}{L}} \cos 2 \pi\left(\frac{x}{L}-\frac{t}{T}\right) \\
& Y_{z}=\frac{h}{2} \frac{\sinh \frac{2 \pi}{L}(d+z)}{\sinh \frac{2 \pi d}{L}} \sin 2 \pi\left(\frac{x}{L}-\frac{t}{T}\right)
\end{aligned}
$$


Here, $z$ is negative measured downward from the still-water surface $(z=-d$ at the bottom). The displacements are out of phase by $T / 4$ in time and $L / 4$ in the direction of motion. The wave form is sinusoidal and applies to waves of small amplitude only.

The full amplitude of oscillation expressed as a fraction of the height from trough to crest is

Horizontal:

$$
\frac{a}{h}=\frac{\cosh \frac{2 \pi}{L}(d+\pi)}{\sinh \frac{2 \pi d}{L}}
$$

Vertical:

$$
\frac{b}{h}=\frac{\sinh \frac{2 \pi}{L}(d+z)}{\sinh \frac{2 \pi d}{L}}
$$

At the surface, $z=0, \frac{d_{s}}{h}=\operatorname{cotl} 1 \frac{2 \pi d}{L}$ and $\frac{b_{s}}{h}=1$.

Figures 3 and 4 show the values of equations 5 and 6 with $\frac{d}{L}$ as the parameter. As $\frac{d}{L}$ approaches zero, the horizontal displacements approach uniformity while the vertical displacements increase linearly from bottom to surface. These circumstances permit a simplification in the theory of long periodic waves which, however, does not alter the fact that they are merely a limiting case of oscillatory wave motion.

Equation 5 can be converted to another form by expressing the hyperbolic functions in terms of exponentials as follows:

$$
\frac{a}{h}=\frac{e^{\frac{2 \pi d}{L}} \cdot e^{\frac{2 \pi z}{L}}+e^{-\frac{2 \pi d}{L}} \cdot e^{-\frac{2 \pi z}{L}}}{e^{\frac{2 \pi d}{L}}-e^{-\frac{2 \pi d}{L}}}
$$

For very large depths, $d \rightarrow \infty$ and $e^{-\frac{2 \pi d}{L}} \rightarrow 0$ giving

$$
\frac{a}{h}=e^{\frac{2 \pi z}{L}}
$$

The quantity $z$ is again negative measured downward from the surface. The same result is obtained for $\frac{b}{h}$ and the paths traced out by fluid elements are circles.

The ratio of the vertical to horizontal amplitudes of the surface orbits is then:

$$
\frac{b_{s}}{a_{s}}=\tanh \frac{2 \pi d}{L}=\frac{e^{\frac{4 \pi d}{L}}+1}{e^{\frac{4 \pi d}{L}}-1}
$$


Equation 2 for the velocity of a wave may be written then:

$$
C=\sqrt{\frac{b_{s}}{a_{s}} \cdot \frac{g L}{2 \pi}}
$$

The fact that the wave velocity depends, in general, upon the wave length eliminates the possibility of building up the characteristics of

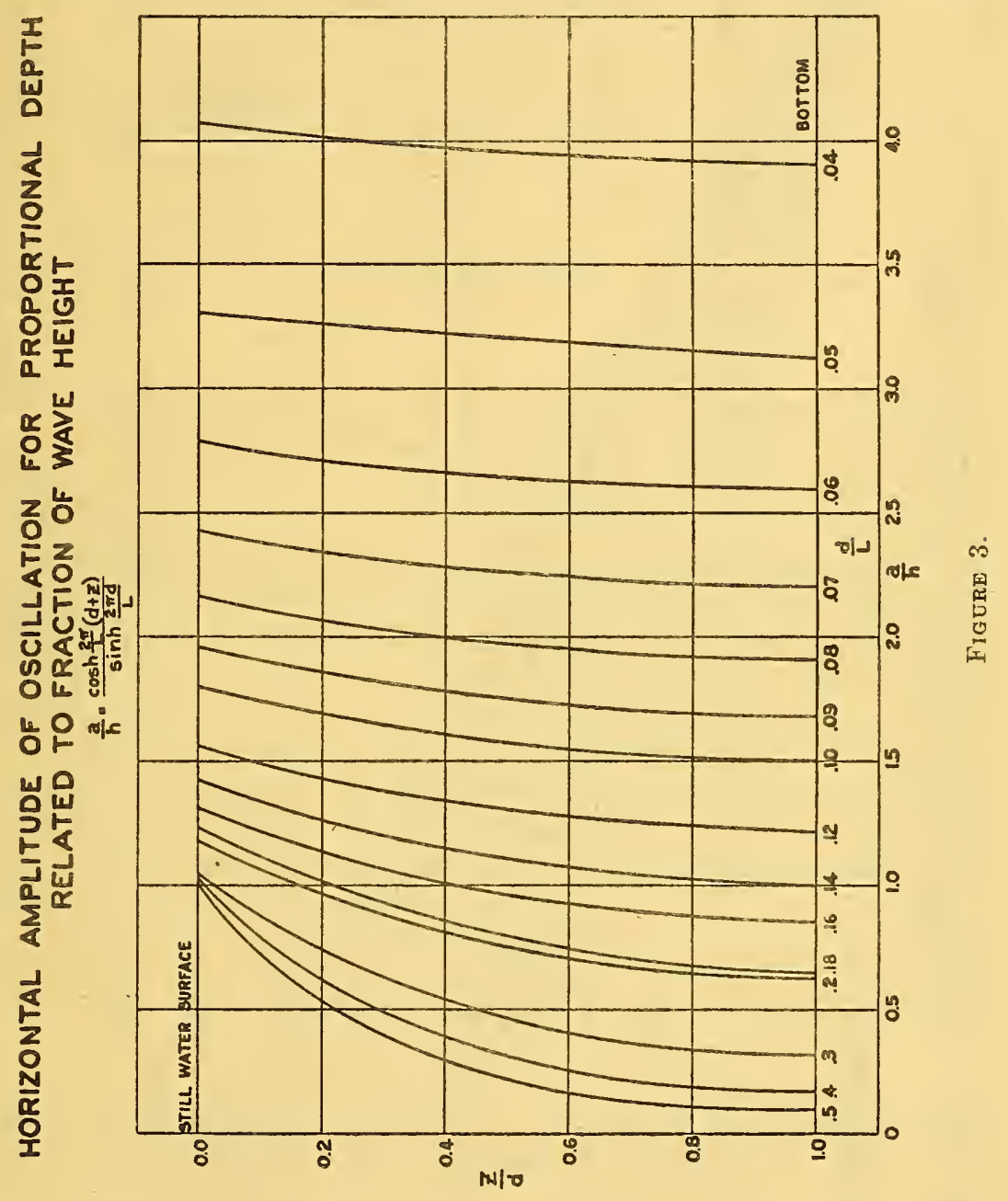

a wave of any form as the summation of the characteristics of waves of different length and amplitude. Each wave length has a characteristic velocity, and superposition of different lengths would give a constantly changing wave form whereas waves observed in nature appear to preserve their identity, at least for distances which are large in comparison to one wave length.

The horizontal and vertical components of the velocity of water 


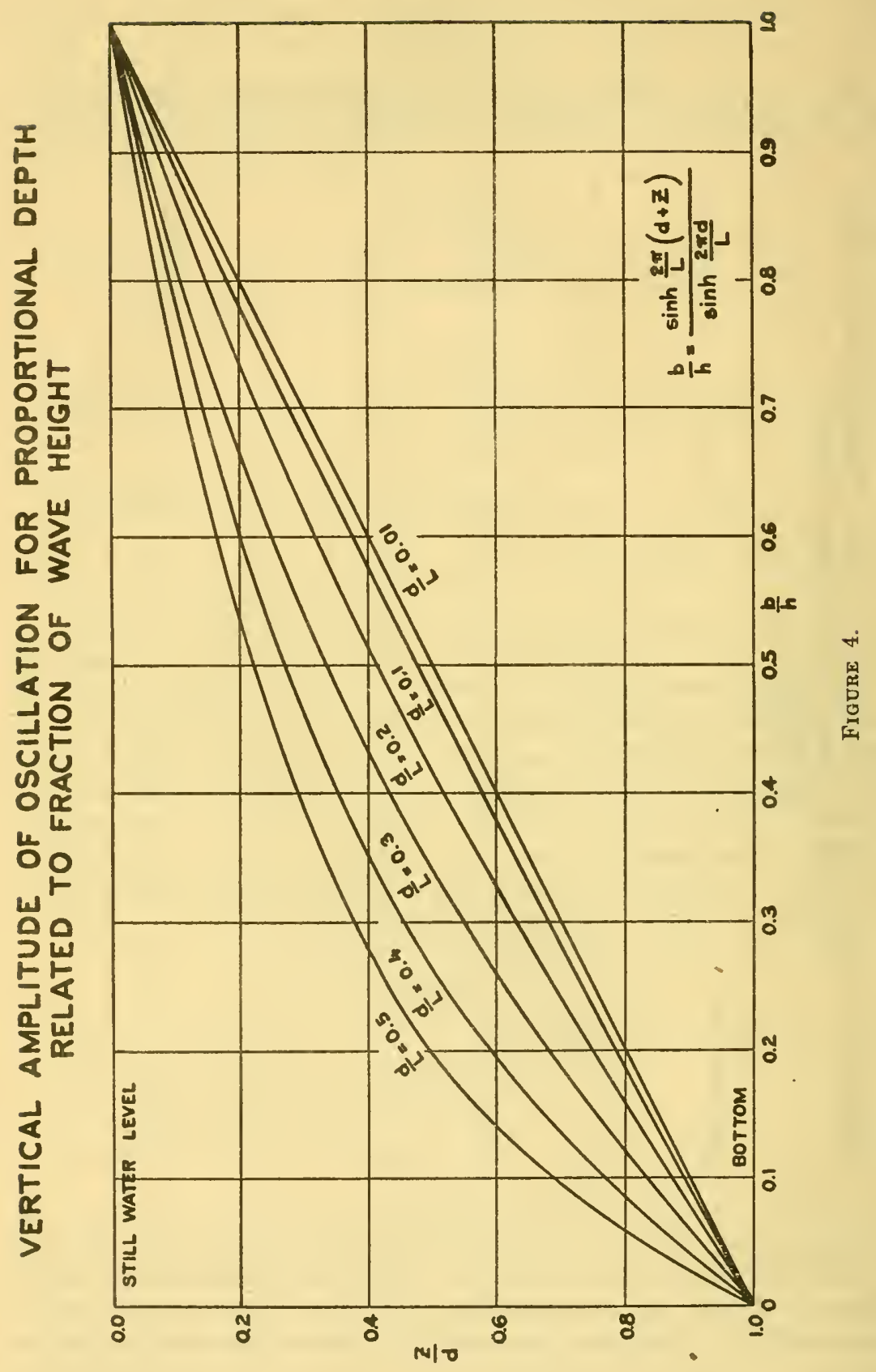


particles occupying an average position at distance $z$ below the surface are obtained by differentiating the displacements with respect to $t$.

$$
\begin{gathered}
U_{z}=\frac{\partial X_{2}}{\partial t}=\frac{\pi a}{T} \sin 2 \pi\left(\frac{x}{L}-\frac{t}{T}\right) \\
W_{2}=\frac{\partial Y_{2}}{\partial t}=-\frac{\pi b}{T} \cos 2 \pi\left(\frac{x}{L}-\frac{t}{T}\right)
\end{gathered}
$$

The average velocities over one-half cycle are then:

$$
U_{z_{a v e}}= \pm \frac{2 a}{T} \quad W_{z_{a v e}}= \pm \frac{2 b}{T}
$$

and the maximum orbital velocities are:

$$
U_{z_{\max }}=\frac{\pi a}{T} \quad W_{z_{\max }}=\frac{\pi b}{T}
$$

In these equations, $a$ and $b$ are the full amplitudes, while $T$ is the period of one full cycle. Computation of these velocities for particular values of $h, T$, and $d$, are effected by equations 5 and 6 or figures 3 and 4, using the value of $L$ for the specified $d$ and $T$ from figure 2 .

These theoretical equations apply only to waves of small amplitude passing over water of constant depth but "the numerical results show that a variation in the depth will have no appreciable influence, provided that the depth everywhere exceeds (say) half the wave length" (5).

At any instant the total energy per unit width of crest in one wave length is obtained as the sum of the potential and kinetic energies. The kinetic energy is the summation from bottom to surface of the individual kinetic energies corresponding to the orbital velocities as given by equations $9 a$ and $9 b$.

The potential energy is computed (6) from the elevation or depression above the still water surface as:

$$
E_{p}=w \int_{x=0}^{x=L} \int_{z=0}^{z=-y} z d z d x
$$

Integrating with respect to $z$ under the surface curve, where $y$ is the particular value of $z$ at the free surface, we obtain:

$$
E_{p}=\frac{w}{2} \int y^{2} d x
$$

For a small wave of sinusoidal form described by the equation:

$$
y=\frac{h}{2} \cos 2 \pi\left(\frac{t}{T}-\frac{x}{L}\right)
$$


the potential energy at any time $t$ per wave length per unit breadth is then:

$$
\begin{aligned}
E_{p} & =\frac{w h^{2}}{8} \int_{x=0}^{x=L} \cos ^{2} \frac{2 \pi x}{L} d x \\
& =\frac{w L h^{2}}{16}
\end{aligned}
$$

The kinetic energy per unit breadth per wave length is obtained by integration of the equation:

$$
E_{k}=\frac{w}{2 g} \int_{x=0}^{x=L} \int_{z=-y}^{z=d}\left(U z^{2}+W z^{2}\right) d x d z
$$

In a deep-water wave of sinusoidal form

$$
E_{k}=\frac{w L h^{2}}{16}=E_{p}
$$

and the total energy is then:

$$
E=E_{k}+E_{p}=\frac{w L h^{2}}{8}
$$

and substituting for $L$ from equation $2 c$

$$
E=0.64 w h^{2} T^{2}
$$

\section{Section 4. WAVES OF FINITE AMPLITUDE}

A. Irrotational theory.-The preceding equations were derived on the assumption that the wave height was very small as compared to the wave length and water depth. However, it is known from experiment that the equations continue to be valid for waves of small but appreciable height. For waves of greater height, theory indicates that certain corrections are necessary. Waves in water of unlimited depth will be considered first.

It has been shown by Stokes that waves of finite amplitude in deep water are not sinusoidal in form and that there is an induced drift or mass transport in the direction of wave motion which modifies the wave velocity and orbital motion. Stokes' (2) conclusions are:

I find that the expression for the velocity of propagation is independent of the height of the waves to a second approximation. With respect to the form of the waves, the elevations are no longer similar to the depressions, as is the case to a first approximation, but the elevations are narrower than the hollows, and the height of the former exceeds the depth of the latter.

There is one result of a second approximation which may possibly be of practical importance. It appears that the forward motion of the particles is not altogether compensated by their backward motion; so that, in addition to their motion of oscillation, the particles have a progressive motion in the direction of propagation of the waves.

The waves produced by the action of the wind on the surface of the sea do not probably differ very widely from those which have just been considered, and which may be regarded as the typical form of oscillatory waves.

If the pressure of the air on the posterior parts of the waves is greater than on the anterior parts, in consequence of the wind, as unquestionably it must be, it is easy to see that some progressive motion must be produced. 
The analysis by Stokes involved a series solution which was shown to be convergent for "deep-water waves" by Levi Civita (7) and for "shallow-water waves" by Struik (8). To a second order of approximation, Stokes found the wave velocity, for any depth of water, to be given by:

$$
C^{2}=\frac{g L}{2 \pi} \frac{e^{\frac{2 \pi d}{L}}-e^{-\frac{2 \pi d}{L}}}{e^{\frac{2 \pi d}{L}}+e^{-\frac{2 \pi d}{L}}}
$$

The equation of the surface of the wave is:

$$
y=a \cos \frac{2 \pi x}{L}+\frac{\pi a^{2}}{L} \cos \frac{4 \pi x}{L} \frac{\left(e^{\frac{2 \pi d}{L}}+e^{-\frac{2 \pi d}{L}}\right)\left(e^{\frac{4 \pi d}{L}}+e^{-\frac{4 \pi d}{L}}+4\right)}{2\left(e^{\frac{2 \pi d}{L}}-e^{\left.-\frac{2 \pi d}{L}\right)^{3}}\right.}
$$

or

$$
y=a \cos m x+K a^{2} \cos 2 m x
$$

where:

$$
m=\frac{2 \pi}{L} ; K=\frac{\pi}{L} \frac{\left(e^{\frac{2 \pi d}{L}}+e^{-\frac{2 \pi d}{L}}\right)\left(e^{\frac{4 \pi d}{L}}+e^{-\frac{4 \pi d}{L}}+4\right)}{2\left(e^{\frac{2 \pi d}{L}}-e^{-\frac{2 \pi d}{L}}\right)^{3}} ; \text { and } a=\frac{h}{2}
$$

The velocity of forward transport (also known as "mass transport") of a particle at a depth $d$ below the still water surface is:

$$
\bar{U}=\frac{4 \pi a^{2}}{L^{2}} \cdot C \cdot \frac{e^{\frac{4 \pi}{L}(z+d)}+e^{-\frac{4 \pi}{L}(z+d)}}{\left(e^{\frac{2 \pi d}{L}}-e^{-\frac{2 \pi d}{L}}\right)^{2}}
$$

The height between trough and crest is $2 a$. The midheight between trough and crest is $K a^{2}$ above $y=0$. Meqsured along the level $y=0$ (still water surface), the portion of the wave length above the still water surface is $\frac{L}{2}-\frac{a K L}{2 \pi}$ while the portion below is $\frac{L}{2}+\frac{a K L}{2 \pi}$. For any value of $L, K$ increases as the depth decreases, producing sharpened crests and flattened troughs. The surface curves (equation 13) for different values of $\frac{d}{L}$ and $\frac{a}{L}$ appear in figure 5 .

When the water depth is great in comparison to the length of the wave, these equations simplify to

$$
\text { Wave velocity: } C=\sqrt{\frac{g \bar{L}}{2 \pi}}
$$

$$
\text { Wave surface: } y=a \cos \frac{2 \pi x}{L}+\frac{\pi a^{2}}{L} \cos \frac{4 \pi x}{L}
$$

Mass transport velocity: $\bar{U}=\frac{4 \pi a^{2}}{L^{2}} e^{\frac{4 \pi z}{L} \cdot C}$

At $x=0$, and $x=L, y=a+\frac{\pi a^{2}}{L} ;$ while at $x=\frac{L}{2}, y=-a+\frac{\pi a^{2}}{L}$ 
The height from trough to crest is $2 a$ and the wave is symmetrical about the crests. The mean elevation between trough and crest is $\frac{\pi a^{2}}{L}$ above the still water level $y=0$. The equation for the wave

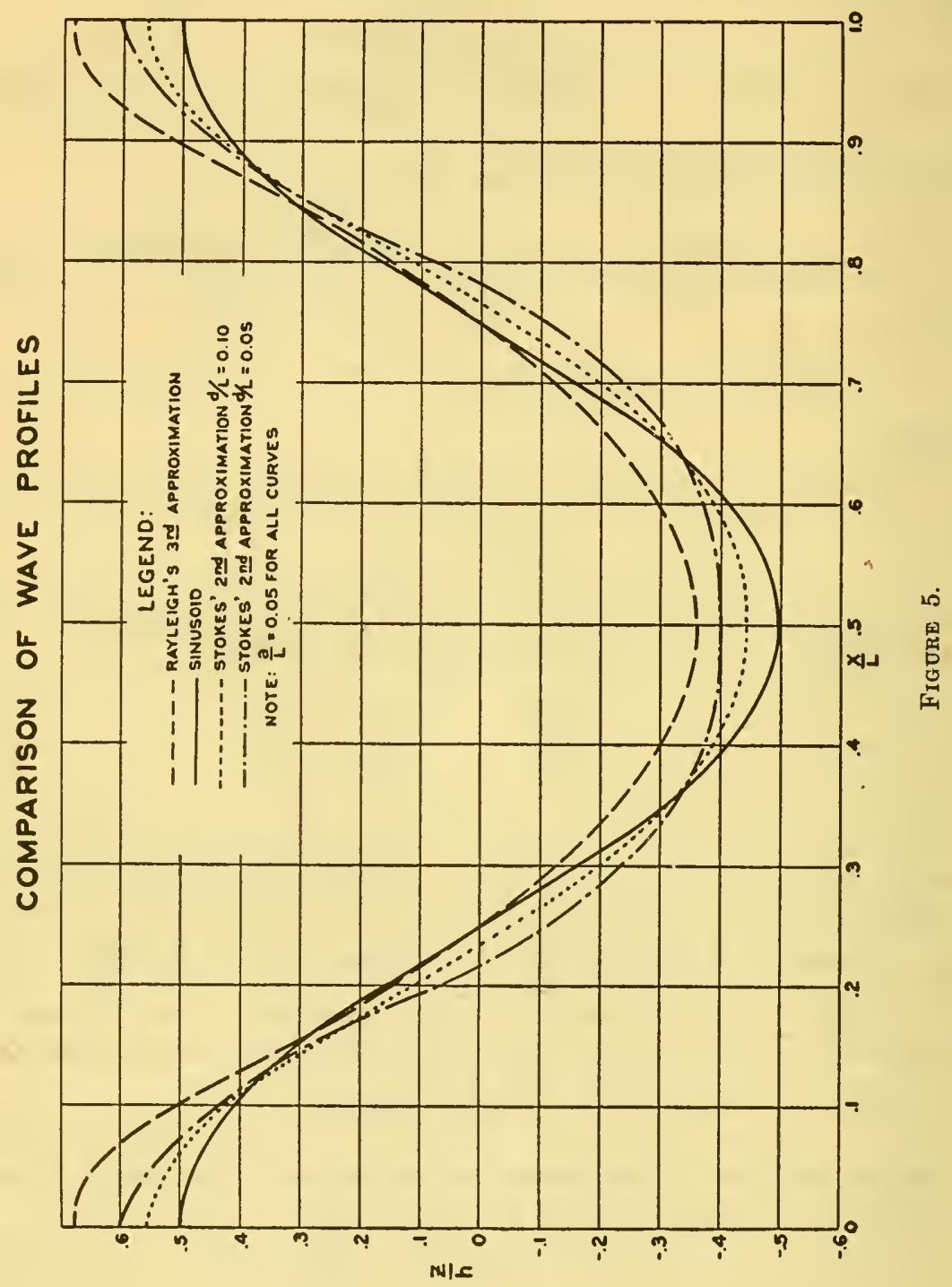

form is also sometimes expressed as

$$
y^{\prime}-\frac{\pi a^{2}}{L}=a \cos \frac{2 \pi x}{L}+\frac{\pi a^{2}}{L} \cos \frac{4 \pi x}{L}
$$

Here $\mathrm{y}^{\prime}$ is the distance above or below a horizontal line at midheight 
between trough and crest. The surface curve cuts the level $y=0$ (equation $13 b$ ) at

$$
x=\frac{L-a}{4} \text { and } x=\frac{3 L+a}{4}
$$

Rewriting equation $14 a$ in terms of $h$ and substituting from equation $12 a$, for $C$, the rate of drift or mass transport at a distance $z$ below the surface is given as:

$$
\bar{U}=h^{2} e^{\frac{4 \pi z}{L}} \sqrt{\frac{g \pi^{3}}{2 L^{3}}}
$$

Here, $z$ is negative when measured downward from the surface.

\section{VELOCITY DISTRIBUTION OF MASS TRANSPORT IN A CLOSED CHANNEL}

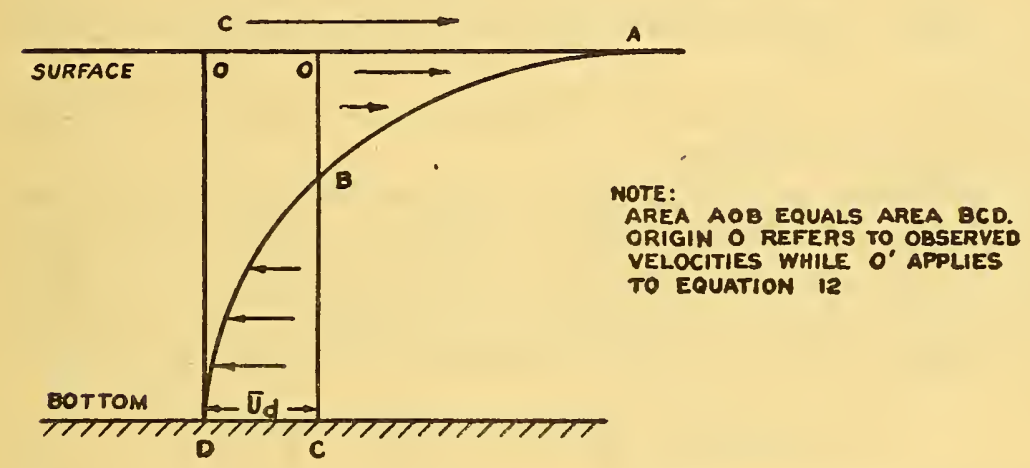

Figure 6.

Integrating this equation between 0 and $-\infty$, we obtain as the total volume transfer per unit width of wave crest

$$
G=h^{2} \sqrt{\frac{g \pi}{32 L}}
$$

The average velocity of drift down to some depth $d$ (which must exceed $L / 2$ if the theory is to apply) is

$$
\bar{U}_{d}=\frac{G}{d}=\frac{h^{2}}{d} \sqrt{\frac{g \pi}{32 L}}
$$

$\bar{U}_{d}$ is approximately the magnitude of the undertow or return flow along the bottom which must exist in a channel closed at the end toward which the waves travel. The observed velocity distribution would be as shown in figure 6 .

If the waves are set up in a closed tank, the velocity distribution in equation $14 b$ must be compensated for a reverse motion which 
reduces the net flow to zero. Equation $14 b$ describes the motion relative to the fluid and may be assumed as superimposed on a uniform return current of velocity as given by equation 14d. In such a closed basin, the observed velocity distribution relative to a fixed point then would be:

$$
\bar{U}_{c}^{\prime}=h^{2} \sqrt{\frac{g \pi}{32 L}}\left[e^{\frac{4 \pi z}{L}} \sqrt{\frac{16 \pi^{2}}{L^{2}}}-\frac{1}{d}\right]
$$

The velocity distributions corresponding to equations $14 b$ and $14 e$ are illustrated in figure 6 . Figure 7 shows the velocity distribution for a wave one foot high and with various periods in a channel of unlimited length. The velocity distribution for waves of other heights is obtained by multiplying by $h^{2}$.

Provided that the depth is of such magnitude that the velocity at the bottom given by equation $14 b$ is negligible, $\bar{U}_{\mathrm{d}}$ in equation $14 d$ is the actual return velocity at the bottom in a channel with closed end. Figure 8 shows this velocity as a function of depth and wave period.

Mitchim (10) found experimentally that equations $14 b$ and $14 d$ represent actual conditions.

The preceding equations apply under conditions of finite wave height; however, the series solution of Stokes includes only the first terms and therefore the equations are theoretically accurate only if the height is small as compared with the length. For deep-water waves, Stokes found as the third approximation to the wave form

$$
y=d \cos \frac{2 \pi x}{L}+\frac{\pi a^{2}}{L} \cos \frac{4 \pi x}{L}+\frac{3 \pi^{2} a^{3}}{2 L^{2}} \cos \frac{6 \pi x}{L}
$$

and for the wave velocity:

$$
C_{h}=\sqrt{\frac{g L}{2 \pi}}\left(1+\frac{2 \pi^{2} a^{2}}{L^{2}}\right)
$$

Equation 16 is represented in figure 9. Regarding equation 15, Stokes notes, "It is remarkable that this equation coincides with that of the prolate cycloid if the latter equation be expanded according to ascending powers of the distance of the tracing point from the center of the rolling circle and the terms of the fourth order are omitted." The coincidence of the surface described by equation 15 with the cycloid is of interest in connection with the trochoidal theory to be discussed later.

To the fourth order of approximation, Stokes obtains as the surface profile

$$
\begin{aligned}
y=a \cos \frac{2 \pi x}{L}- & \left(\frac{1}{2} m a^{2}+\frac{17}{24} m^{3} a^{4}\right) \cos \frac{4 \pi x}{L}+\frac{3}{8} m^{2} a^{3} \cos \frac{6 \pi x}{L} \\
& -\frac{1}{3} m^{3} a^{4} \cos \frac{8 \pi x}{L}+\ldots
\end{aligned}
$$




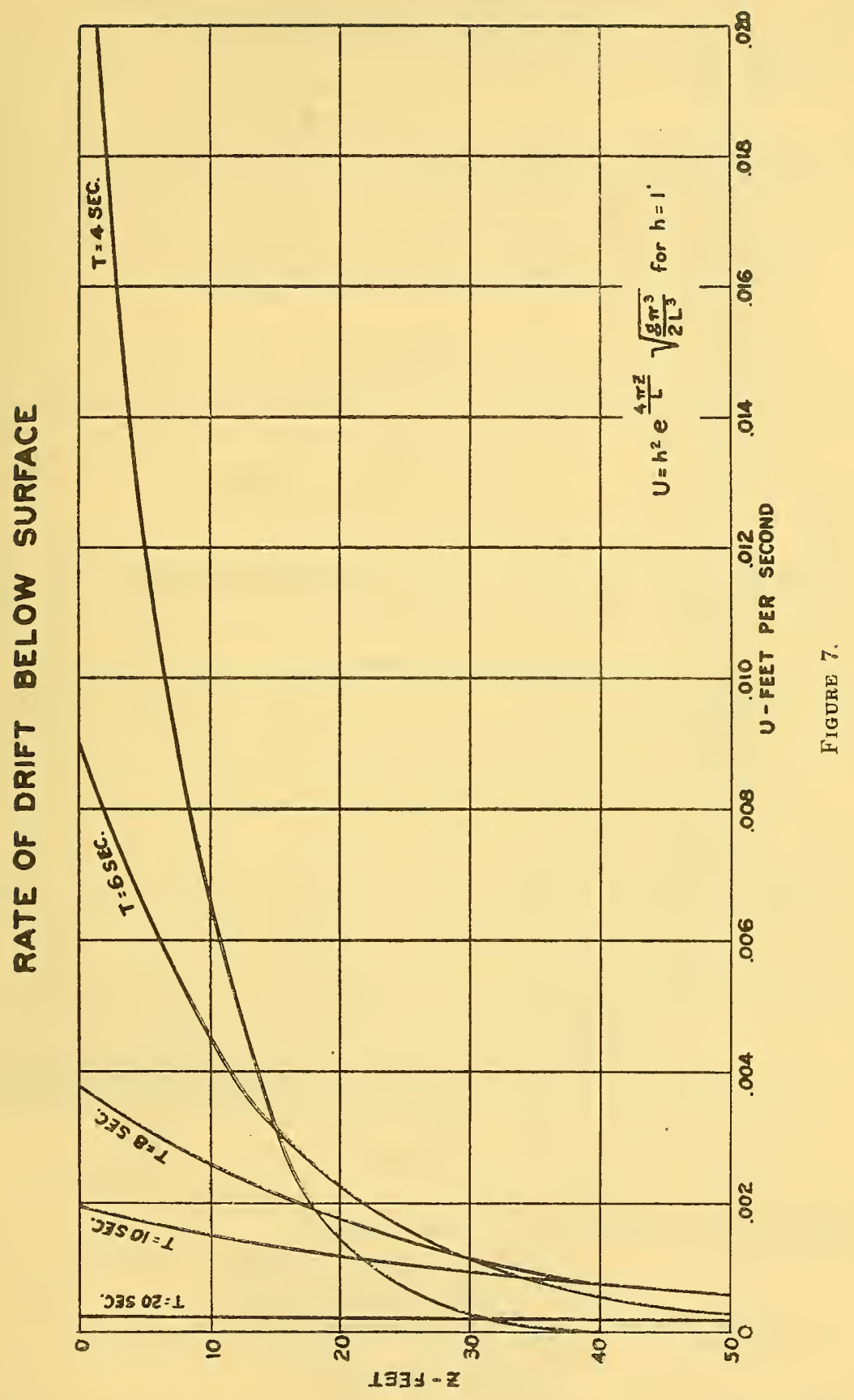


BOTTOM RETURN VELOCITY IN TERMS

OF WAVE PERIOD AND DEPTH

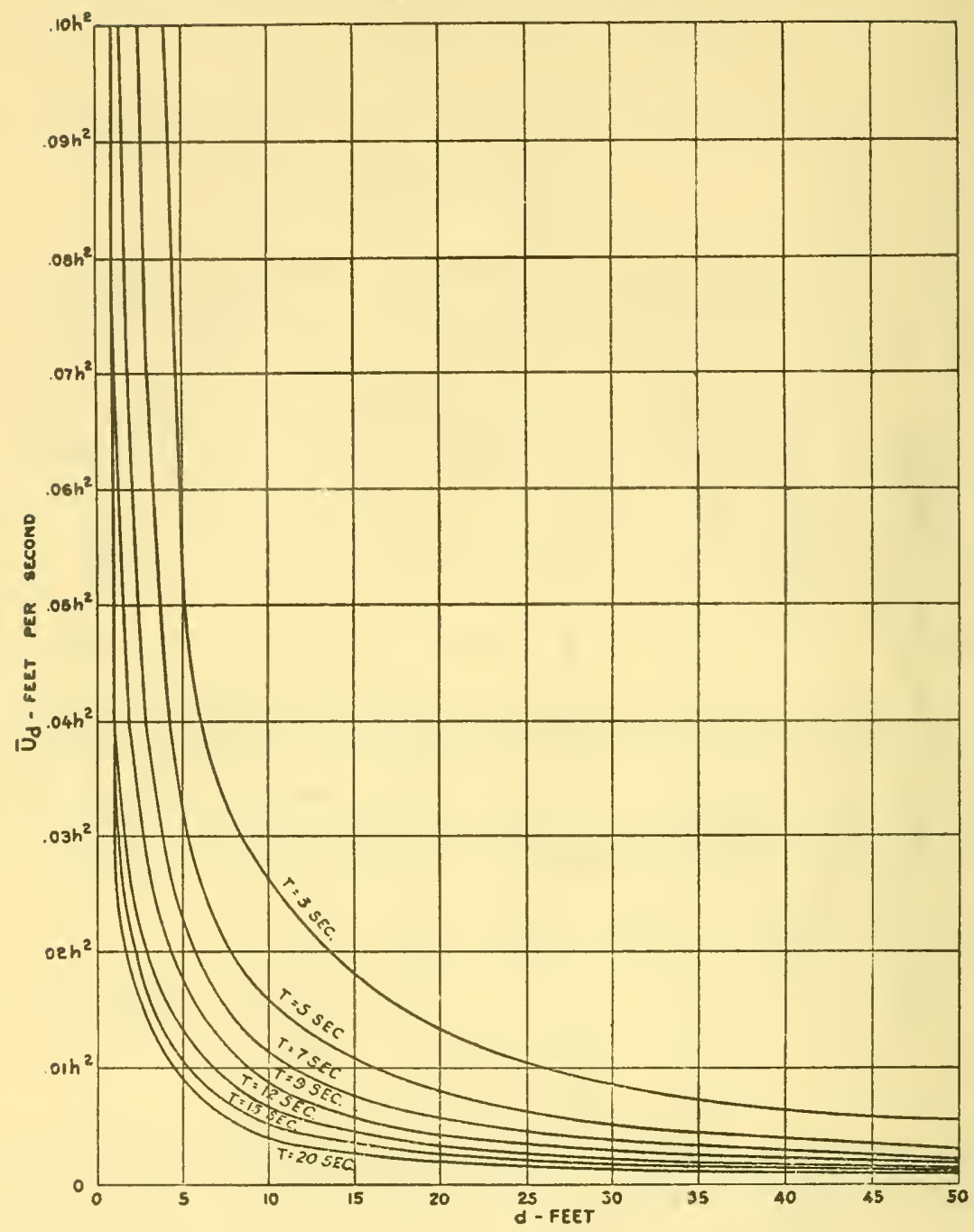

FIGURE 8.

and as the expression for the wave velocity:

$$
\begin{aligned}
C_{h} & =\sqrt{\frac{g L}{2 \pi}\left(1+\frac{4 \pi^{2} a^{2}}{L^{2}}+\frac{20 \pi^{4} a^{4}}{L^{4}}\right)} \\
& =\sqrt{\frac{g L}{2 \pi}\left(1+\alpha^{2}+\frac{5}{4} \alpha^{4}\right)}
\end{aligned}
$$

and:

$$
\left(\frac{C_{h}}{C}\right)^{2}=1+\alpha^{2}+\frac{5}{4} \alpha^{4} \ldots
$$


This equation does not agree with the trochoid to the same order of approximation, the difference in ordinates being $-\frac{2}{3} a^{4} \cos 2 x$ the surface curve lying "a little above the trochoid at the trough and a little below it at the shoulders" (2).

Levi-Civita (7) showed that Stokes' series was convergent and confirmed the surface curve of equation 17 for waves in decp water. For the velocity of the wave, to the fifth approximation, he obtained

where

$$
C_{h}^{2}=\frac{g L}{2 \pi}\left(1+\alpha^{2}-\alpha^{3}+\frac{7}{4} \alpha^{4}-\frac{49}{12} \alpha^{5}+\ldots\right)
$$

$$
\alpha=f(h)
$$

or expressing $C_{h}$ in terms of $h$ and $L$ the foregoing equation takes the form

$$
C_{h}^{2}=\frac{g L}{2 \pi}\left(1+\frac{\pi^{2} h^{2}}{L^{2}}+\frac{\pi^{4} h^{4}}{2 L^{4}}+\ldots\right)
$$

Figure 9 shows the correction for finite wave height. The fact that the series is convergent demonstrates, at least theoretically, the possibility of periodic waves of permanent form.

Regarding the form of the waves, Stokes remarked:

It appears also that, whatever be the order of approximation the waves will be symmetrical with respect to vertical planes passing through their ridges, as also with respect to vertical planes through their lowest points.

This conclusion was also confirmed by Levi-Civita.

When the depth of the water is about the same magnitude as the length of the wave, or less, the terms involving the water depth in the equations must be retained. The analysis employed in this case is similar to that followed for the case of deep water but the solution is somewhat more complicated. Stokes (2) and Struik (8) both obtained solutions to a third approximation. The equations will be given here without derivation.

The expression for the velocity of propagation of the wave is:

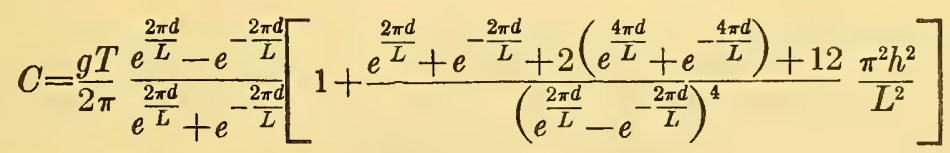

The equation of the surface of the wave is:

$$
\begin{gathered}
y=a \cos \frac{2 \pi x}{L}-\frac{2 \pi a^{2}}{L} \frac{\left(e^{\frac{2 \pi d}{L}}+e^{\left.-\frac{2 \pi d}{L}\right)\left(e^{\frac{4 \pi d}{L}}+e^{-\frac{4 \pi d}{L}}+4\right)}\right.}{\left(e^{\frac{2 \pi d}{L}}-e^{-\frac{2 \pi d}{L}}\right)^{4}} \cos \frac{4 \pi x}{L} \\
+\frac{\pi^{2} a^{3}}{L^{2}} \frac{5\left(e^{\frac{12 \pi d}{L}}+e^{-\frac{12 \pi d}{L}}\right)+14\left(e^{\frac{8 \pi d}{L}}+e^{-\frac{8 \pi d}{L}}\right)+19\left(e^{\frac{4 \pi d}{L}}+e^{-\frac{4 \pi d}{L}}\right)}{\left(e^{\frac{2 \pi d}{L}}-e^{-\frac{2 \pi d}{L}}\right)^{6}} \cos \frac{6 \pi x}{L}
\end{gathered}
$$




\section{RATIO OF WAVE VELOCITIES INDICATING THE EFFECT OF FINITE HEIGHT OF WAVE}

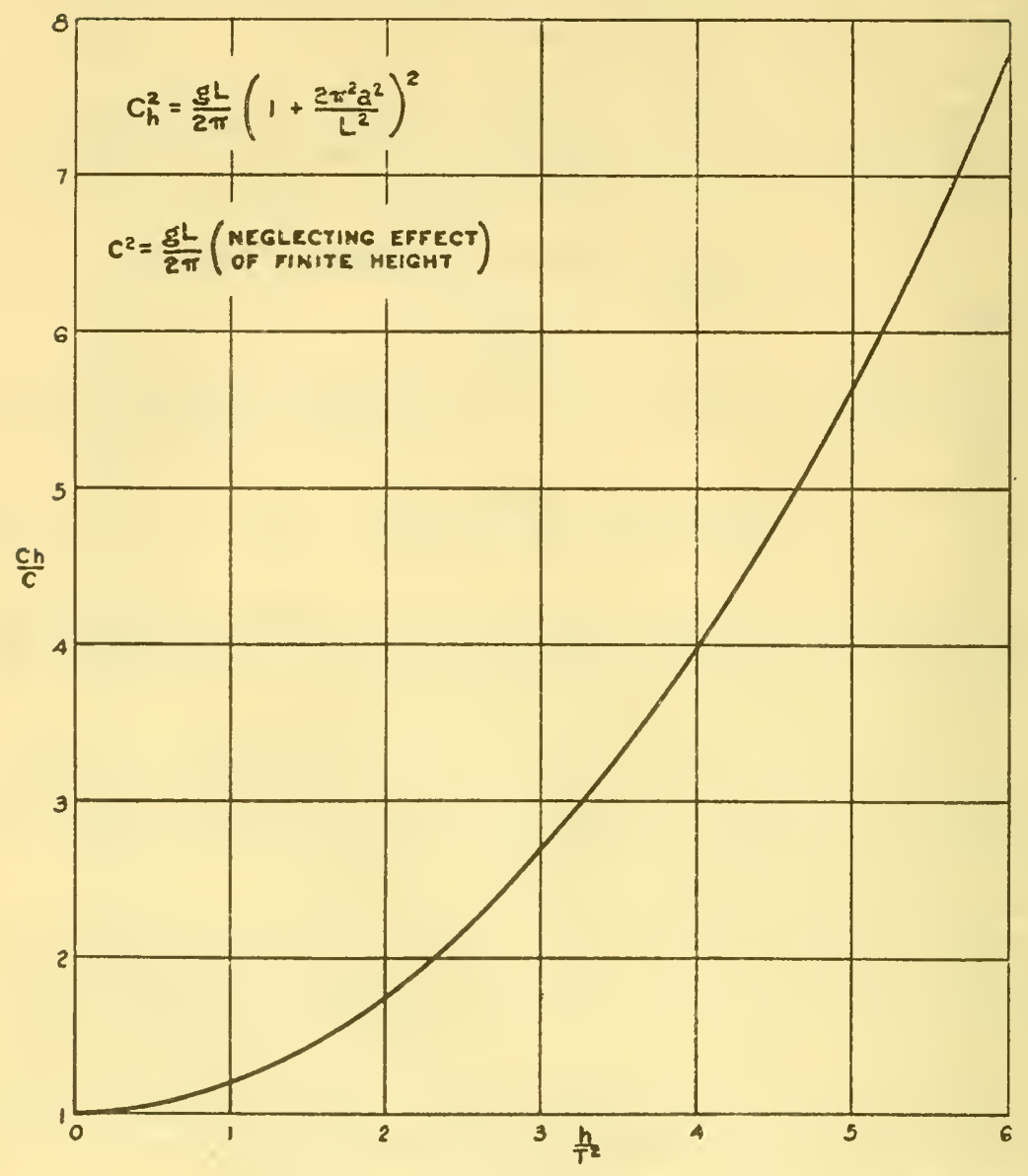

Figure 9.

\section{Stokes (2) notes:}

The disadvantage of the approximation for the case of a finite as compared with that of an infinite depth is not however quite so great as might at first sight appear. There can be little doubt that in both cases alike the series cease to be convergent when the limiting wave, presenting an edge of $120^{\circ}$, is reached. In the case of an infinite depth, the limit is reached for some determinate ratio of the height of a wave to the length, but clearly the same proportion could not be jreserved when the depth is much diminished. In fact, high oscillatory waves in shallow water tend to assume the character of a series of disconnected solitary waves, and the greatest possible height depends mainly on the depth of the fluid, being but little influenced by the length of the waves, that is, the distance from crest to crest. To make the comparison fair therefore between the convergency of the series in the cases of a finite and of an infinite depth, we must not suppose the coefficient of $\cos i n \phi / C^{\prime}$ the same in the two cases for the same length of wave, 
but take it decidedly smaller in the case of the finite depth, such for example as to bear the same proportion to the greatest possible value in the two cases.

But with all due allowance to this consideration, it must be confessed that the approximation is slower in the case of a finite depth. That it must be so is seen by considering the character of the developments, in the two cases, of the ordinate of the profile in a harmonic series in terms of the abscissa, or of a quantity having the same period and the same mean value as the abscissa. The flowing outline of the profile in deep water lends itself readily to expansion in such a series. But the approximately isolated and widely separated elevations that represent the profile in very shallow water would require a comparatively large number of terms in their expression in harmonic series in order that the form should be represented with sufficient accuracy. In extreme cases the fact of the waves being in series at all has little to do with the character of the motion in the neighbourhood of the elevations, where alone the motion is considerable, and it is not therefore to be wondered at if an analysis essentially involving the length of a wave should prove inconvenient.

The problem of the greatest possible height attainable by any given oscillatory wave of permanent form has been considered by a number of investigators. Stokes analyzed the problem on the basis of a velocity superimposed on the water which would bring the wave to rest. The flow being assumed as frictionless, Bernoulli's equation applies and the greatest possible height is attained when the velocity at the crest is zero. Writing a potential function for flow in the immediate vicinity of the crest, we have:

$$
\phi=A r^{n} \sin n \theta
$$

The profile is symmetrical about the crest and only sine terms are admissible. Since no flow occurs perpendicular to the surface, then:

$$
\frac{\partial \phi}{\partial \theta}=0=-A r^{n} \cdot n \cos n \theta
$$

and

$$
\frac{\partial \phi}{\partial r}=n A \sin n \theta r^{n-1}=V=\sqrt{2 g r \cos \theta}
$$

Comparing the exponents of $r$

$$
\begin{aligned}
n-1 & =\frac{1}{2} \text { and } n=\frac{3}{2} \\
\cos n \theta & =0 \text { and } \frac{3}{2} \theta=\frac{\pi}{2}
\end{aligned}
$$

therefore

$$
\theta=\frac{\pi}{3}
$$

The complete angle is then $2 \pi / 3$ or $120^{\circ}$. Concerning this result, Stokes (2) remarks:

This, however, leaves untouched the question whether the disturbances can actually be pushed to the extent of yielding crests with sharp edges . . . After careful consideration, I feel satisfied that there is no such earlier limit, but that 
we may actually approach as near as we please to the form in which the curvature at the vertex becomes infinite, and the vertex becomes a multiple point where the two branches, with which alone we are concerned, enclose an angle of $120^{\circ}$.

Michell (11) found theoretically that the limiting height from trough to crest is $0.14 L$. Havelock (12) obtained as the maximum value $0.1418 L$. Quoting Stokes further on the subject:

There can be little doubt that in both cases (deep water and shallow water) alike the series cease to be convergent when the limiting wave, presenting an edge of $120^{\circ}$, is reached.

In fact, high oscillatory waves in shallow water tend to assume the character of a series of disconnected solitary waves, and the greatest possible height depends mainly on the depth of the fluid, being but little influenced by the length of the waves, that is, the distance from crest to crest.

The flowing outline of the profile in deep water lends itself readily to expansion in series. But the approximately isolated and widely separated elevations that represent the profile in very shallow water would require a comparatively large number of terms in their expression in harmonic series in order that the form should be represented with sufficient accuracy.

Rayleigh (13) compared the potential and kinetic energies of waves which are not infinitely small and found that the kinetic energy exceeds the potential but the error is small.

These equations for a stable wave form were obtained for conditions of constant atmospheric pressure on the free surface. Waves moving under the influence of strong winds may well exhibit different forms; but laboratory experiments on water waves under still air confirm the theoretical equations.

B. Trochoidal theory.-Classical theory concludes that the surface profile of a wave of finite height is approximately trochoidal, and measurements of natural and model waves conform approximately to this type of curve in which the crests become sharper and the troughs flattened as the height increases in proportion to the length. Experiment has not yet conclusively shown whether or not surface water waves are exactly trochoidal but the deviations therefrom are small. This fact was made the basis for the trochoidal theory of deep-water waves first proposed by Franz Gerstner (14) and developed independently much later by Rankine (15). The outstanding features of this theory are that: the flow is rotational and therefore cannot be generated from rest by conservative forces, and the wave movement is not accompanied by a mass transport in the direction of wave travel.

Stokes' (2) comments on this theory are of particular interest and will be quoted in some detail:

This case of motion has latterly attracted a good deal of attention, partly no doubt from the facility of dealing with it, but partly it would seem, from misconceptions as to its intrinsic importance.

Expressing the position of a particle at time $t$ in terms of its initial 
coordinates $x_{0}$ and $y_{0}$ and including two constants $m$ and $C$ and a parameter $K$ which is a function of $y$ only; then:

$$
\begin{aligned}
& x=x_{0}+K \sin m\left(x_{0}-C t\right) \\
& y=y_{0}+K \cos m\left(x_{0}-C t\right)
\end{aligned}
$$

It will be easily seen that these equations, regarded merely as expressing the geometrical motion of points, and apart from the physical possibility of the motion, represent a wave disturbance of periodic character traveling in the direction $0 x$ with a velocity of propagation $C$.

Assuming infinite depth and applying the requirements of continuity and constant pressure on the surface, Stokes shows that the displacements must decrease exponentially with distance below the surface; and that the wave velocity for all waves (small and finite height) is given by

$$
C^{2}=\frac{g L}{2 \pi}
$$

Furthermore, if the motion is reduced to steady flow by superimposing a velocity $-C$ on the entire liquid mass, the stream lines, designated by $y_{o}=$ constant, are also lines of constant pressure.

This is undoubtedly no necessary property of wave motion converted into steady motion, which only requires that the particular stream line at the surface shall be one for which the pressure is constant, though Gerstner has expressed himself as if he supposed it necessarily true; it is merely a character of the special case investigated by Gerstner and Rankine. Nevertheless, in the case of deep water it must be very approximately true.

In any given case of wave motion, the flow which remains when the waves have been caused to subside in the manner above explained is easily determined, since we know that in the motions of a liquid in two dimensions the angular velocity is not affected by forces applied to the surface.

In Gerstner and Rankine's solution

$$
\begin{aligned}
\text { vorticity } & =2 \omega=\frac{\partial V}{\partial x}-\frac{\partial U}{\partial y} \\
\omega & =-\frac{m^{3} a^{2} C e^{-2 m y}}{1-m^{2} a^{2} e^{-2 m y}} \\
U^{\prime} & =-m^{2} a^{2} C e^{-2 m y}
\end{aligned}
$$

where $U^{\prime}=$ horizontal velocity remaining after wave motion has been destroyed.

It appears then that in order that it should be possible to excite these waves in deep water previously free from wave disturbance, by means of pressures applied to the surface, a preparation must be laid in the shape of a horizontal velocity decreasing from the surface downwards according to the value $e^{-2 m y}$, where $y$ is a function of the depth $y^{\prime}$ determined by the transcendental equation of $y$, and moreover, a velocity decreasing downwards according to this law will serve for waves of the present kind of only one particular height depending on the coefficient of the exponential in the expression for the flow.

The oscillatory waves which most naturally present themselves to our attention are those which are excited in the ocean or on a lake by the action of the wind, or those which have been so excited and propagated into (practically, though not in a rigorous mathematical sense) still water. Of the latter kind are the surf 
which breaks upon our western coasts as a result of storms out in the Atlantic, or the grand rollers which are occasionally observed at St. Helena and Ascension Island. The motion in these cases having been produced from rest, by forces applied to the surface, there is no molecular rotation, and therefore the investigation of the present paper strictly applies. Moreover, if we conceive the waves gradually produced by suitable forces applied to the surface, *** the investigation applies to the waves (secular change apart) at any period of their growth, and not merely when they have attained one particular height.

There can be no question, it seems to me, that this is the class of oscillatory waves which on merely physical grounds we should naturally select for investigation. The interest of the solution first given by Gerstner, and it is of great interest, arises not from any physical preeminence of the class of waves to which it relates, but from the imperfection of our analysis, which renders it important to discuss a case in which all the circumstances of the motion can be simply expressed in mathematical terms without any approximation. And though this motion is not exactly that which on purely physical grounds we should prefer to investigate, namely, that in which the molecular rotation is nil, yet unless the height of the waves be extravagant, it agrees so nearly with it that for many purposes the simpler expressions of Rankine may be used without material error, even when we are investigating wave motion of the irrotational kind.

Stokes' criticism appears to be substantiated by the experimentally determined fact that waves induce a mass transport of the magnitude predicted by his theory.

It is interesting to note that the work of Gerstner and Rankine dealt only with waves in deep water. Gaillard's summary of the trochoidal theory is extended to shallow-water waves, apparently by grafting on the trochoidal surface form the conclusions of Stokes regarding the orbital and wave velocities. The antecedents of Gaillard's presentations are not clear from his text but he has been so widely quoted that, regardless of historical background, Gaillard's (16) book today represents the trochoidal theory however he may have arrived at his equations. For ease of reference, all of the important equations of Gaillard will be quoted here with repetitions of previous equations indicated by the equation numbers.

Deep-water waves. - In terms of the method of generating the curve, the trochoidal surface is described by the equations,

$$
\begin{aligned}
& x=R \theta-r \sin \theta \\
& y=R-r \cos \theta
\end{aligned}
$$

Here $R$ is the radius of the rolling circle $(L=2 \pi R)$ and $r$ is the radius of the tracing circle $(h=2 r)$. The positions of crest and trough relative to still-water level are:

$$
\begin{aligned}
& \text { Height of crest }=\frac{h}{2}+0.7854 \frac{h^{2}}{L} \\
& \text { Depth of trough }=\frac{h}{2}-0.7854 \frac{h^{2}}{L}
\end{aligned}
$$


The general shape and method of generation are shown by figure 10 .

The wave velocity for waves of all heights is

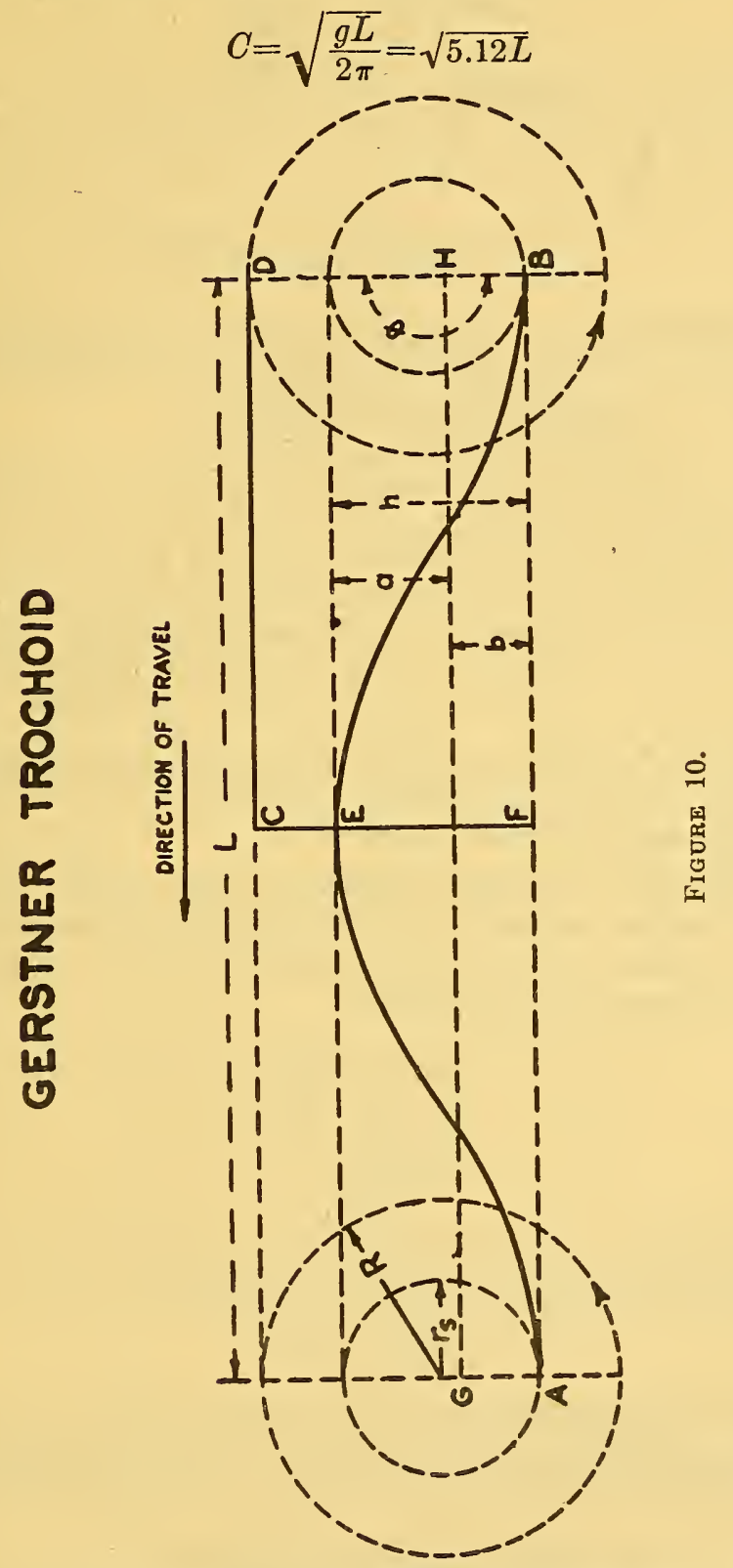

The radii of the orbits below the surface decrease with depth, their values being given by:

$$
\begin{aligned}
r & =a=r_{s} e^{\frac{2 \pi z}{L}} \\
& =\frac{h^{\frac{2 \pi z}{L}}}{2}
\end{aligned}
$$


The total kinetic and potential energy per wave length is

$$
E=\frac{w L h^{2}}{8}\left(1-4.93 \frac{h^{2}}{L^{2}}\right)
$$

Of this amount of energy, one-half is transmitted forward with the wave form and, therefore, the power transmitted per unit of crest width is

$$
P=\frac{E}{2 T}=\frac{w h^{2}}{16} \sqrt{\frac{g L}{2 \pi}}\left(1-4.93 \frac{h^{2}}{L^{2}}\right)
$$

Shallow-water waves.-The orbits of these waves are (16) "ellipses instead of circles, the eccentricity of the ellipses depending upon the ratio of the wave length to the depth of water."

For a given wave length, the eccentricity decreases with an increase of depth, until at depths greater than half a wave length the ellipses are scarcely distinguishable from circles, while in very shallow water they tend to approach right lines.

The orbits decrease in size below the surface, their focal distance remaining constant, the vertical axes therefore decreasing more rapidly relatively than the horizontal, until, at the bottom, were the latter horizontal and frictionless, the vertical axes would become zero, and the particles would move in horizontal straight lines of length equal to the focal distance of the elliptical orbits of the upper particles.

Each particle revolves about the center of the ellipse with an angular velocity which is not constant, but is greater in the vicinity of the crest and trough, and less at midheight than if the orbit were a circle.

These statements of Gaillard have been quoted in full because they described in words, the behaviour mathematically described by equations $4 a, 4 b, 5$, and 6 . The equation for the ratio of semi-minor to semimajor axes of the elliptical orbits is:

$$
\begin{gathered}
\frac{b_{s}}{a_{s}}=\tanh \frac{2 \pi d}{L} \\
=\frac{e^{\frac{4 \pi d}{L}}-1}{e^{\frac{4 \pi d}{L}}+1}
\end{gathered}
$$

The equations describing the reduced surface are:

$$
\begin{aligned}
& x=R \theta-a \cdot \sin \theta \\
& y=b \cos \theta
\end{aligned}
$$

The origin is taken as the orbit center of a crest particle and the surface point fixed as shown in figure 11 . The wave velocity is given by:

$$
C=\sqrt{\frac{b_{s}}{a_{s}} \frac{g L}{2 \pi}}=\sqrt{5.12 \frac{b_{s}}{a_{s}} L}
$$

The total kinetic and potential energy per wave length is:

$$
E=\frac{w L h^{2}}{8}\left(1-19.74 \frac{a_{s}^{2}}{L^{2}}\right)
$$


The ratio $\frac{a_{\mathrm{s}}}{L}$ is a function of $\frac{h}{L}$ and $\frac{d}{L}$,and consequently, the energy may be expressed as

$$
E=\frac{w L h^{2}}{8}\left(1-M \frac{h^{2}}{L^{2}}\right)
$$

The quantity $M$ is shown in figure 12 as a function of $\frac{d}{L}$. It is evident that equation 33 is the limiting case of equation 37 as $\frac{d}{L}$ increases, and that the two equations are identical for $\frac{d}{L}=0.5$.

\section{REDUCED TROCHOID}

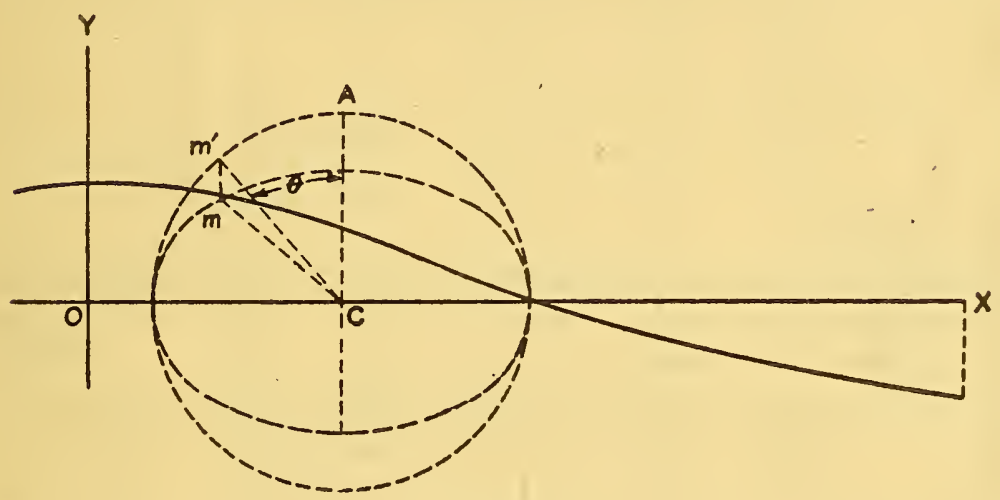

Figure 11.

The power transmitted per unit of crest width is

$$
P=\frac{E}{2 T}=\frac{w h^{2}}{16} \sqrt{\frac{b_{s}}{a_{s}}} \frac{g L}{2 \pi}\left(1-M \frac{h^{2}}{L^{2}}\right)
$$

For both deep-water and shallow-water waves, Gaillard (16) states that the potential energy is transmitted ahead with the wave form. The equations for the power transmitted are based on this relationship.

\section{Section 5. WAVE GROUPS AND TRANSMISSION OF ENERGY}

It is frequently observed that waves break on the shore in "sets," reaching a maximum at regular intervals as, for example, at every seventh wave. There appears to be nothing unique about a beat frequency of seven except that when the number in the group is less, the variation in height is small, and when more waves are involved the interval covered by the passage of the group is too long to be noted by the casual observer. In wave tanks, wave groups may be generated by operating the wave machine through only a few strokes. In both 
situations, the observer can follow a particular wave crest only a finite distance before it disappears. Close observation reveals that the wave group maintains its identity but that individual waves pass

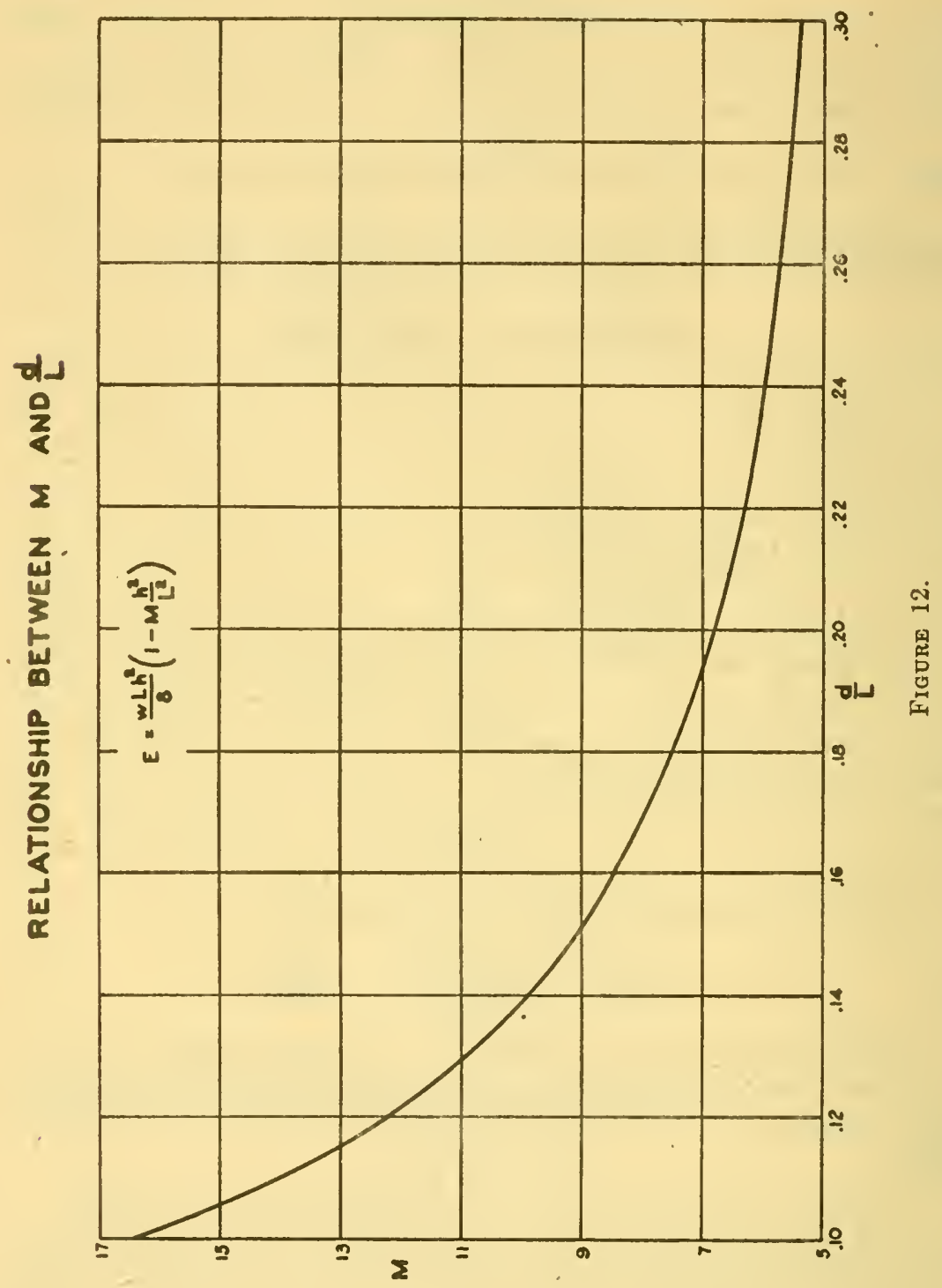

through the group, rising up out of comparatively calm water at the rear, reaching a maximum at the center, and then disappasing at the front of the group. The phenomenon is similar to interference in optics or beat frequency in acoustics.

A small number of waves generated by a wave plunger in a straight tank presents a most interesting phenomenon. If attention is fixed on 
the first wave in the group it is seen to decrease continually in height, finally disappearing altogether in the undisturbed water ahead of the group. The last wave in the group is usually not so clearly defined as the first but, if the plunger is stopped quickly, another wave is seen to develop behind the last wave generated. As the wave group continues through the tank this process is repeated, the leading waves gradually disappearing while new waves develop in the rear. Furthermore, if attention is fixed on any one wave initially at the rear of the group, it will be seen to advance in group position and, if the tank is long enough, finally disappear at the front. The same phenomenon appears in the bow waves of a ship, each wave as it moves diagonally outward loses height and finally disappears while at the rear waves appear which are not attached to the ship.

The reason for this behavior of a group of waves lies in the rate of transmission of energy. The mathematical treatment will be summarized later but an approximately correct physical explanation can be made in simple terms. Assume to be correct Gaillard's statement that half of the wave energy moves forward with the wave form (at the wave velocity). As the first wave in the group advances one wave length, its form induces corresponding velocities in the previously undisturbed water and the kinetic energy corresponding to these velocities must be drawn from the energy flowing ahead with the form. If there is equipartition of energy in the wave, half of the potential energy which advanced with the wave must be given over to the kinetic form and the wave loses height. Advancing another wave length another half of the potential energy is used to supply kinetic energy to the undisturbed liquid. The process continues until the first wave is too small to identify. The second, third, and subsequent waves move into water already disturbed and the rate at which they lose height is less than for the first wave. At the rear of the group, the potential energy might be imagined as moving ahead, leaving a flat surface and half of the total energy behind as kinetic energy. But the velocity pattern is such that flow converges toward one section thus developing a crest and diverges from another section forming a trough. Thus the kinetic energy is converted into potential and a wave develops in the rear of the group. This description is crude. The phenomenon does not proceed in the stepwise fashion described; but the general conclusion is correct, namely, that if the whole wave energy does not move with the wave velocity, the leading wave in the group must lose height and other waves develop at the rear. It is apparent that the group of waves considered as an entity moves more slowly than the waves comprising it at any moment.

If we imagine the entire wave energy to advance with the wave velocity, then energy is being supplied to the undisturbed water ahead 
at a rate sufficient to develop the full wave form in one wave period and all energy is being withdrawn from the water behind in the same period, leaving a horizontal surface and zero motion. That such a situation is difficult to imagine is perhaps an intuitive proof that the full wave energy does not advance with the wave velocity.

Consider next the problem of beat frequeney in natural waves and assume that two infinite wave trains of equal amplitude and very nearly equal wave length are running in the same direction. The elevation of the water surface is the summation of the elevations of the individual waves. Limiting the analysis to waves of small amplitude, which are very nearly sinusoidal, the surface curve would be described by (17)

$$
y=a \sin \left(\frac{2 \pi x}{L_{1}}-\frac{2 \pi t}{T_{1}}\right)+a \sin \left(\frac{2 \pi x}{L_{2}}-\frac{2 \pi t}{T_{2}}\right)
$$

Rewriting the equation in equivalent form,

$$
y=2 a \cos \left\{\pi x\left(\frac{1}{L_{1}}-\frac{1}{L_{2}^{-}}\right)-\pi t\left(\frac{1}{T_{1}}-\frac{1}{T_{2}}\right)\right\} \sin \left\{\pi x\left(\frac{1}{L_{1}}+\frac{1}{L_{2}}\right)-\pi t\left(\frac{1}{T_{1}}+\frac{1}{T_{2}}\right)\right\}
$$

If $L_{1}$ and $L_{2}$ are very nearly equal, the wave form at any time $t$ consists of a series of sine waves of length approximately $L$ and amplitudes which increase from zero to $2 a$ and then decrease to zero. Fixing attention on the surface form, the equation states that the group advances a distance

in a time

$$
\Delta x=\frac{1}{\frac{1}{L_{1}}-\frac{1}{L_{2}}}
$$

$$
\Delta t=\frac{1}{\frac{1}{T_{1}}-\frac{1}{T_{2}}}
$$

The group velocity is then, with $L=C T$

$$
C_{G}=\frac{\Delta x}{\Delta t}=\frac{C_{1} C_{2}\left(T_{2}-T_{1}\right)}{C_{2} T_{2}-C_{1} T_{1}}
$$

Passing to the limit,

$$
C_{G}=\frac{d x}{d t}=\frac{C^{2} d T}{d(C T)}=C-L \frac{d C}{d T}=\text { group velocity }
$$

For waves of small amplitude in any depth of water

and

$$
C=\sqrt{\frac{g L}{2 \pi}+\tanh \frac{2 \pi d}{L}}
$$

$$
C_{G}=\frac{C}{2}\left(1+\frac{\frac{4 \pi d}{L}}{\sinh \frac{4 \pi d}{L}}\right)
$$


Figure 13 shows the magnitude of the ratio $\frac{C_{G}}{C}$ as a function of $\frac{d}{L}$. As $\frac{4 \pi d}{L}$ becomes very small, $\sinh \frac{4 \pi d}{L}=\frac{4 \pi d}{L}$ and $\frac{C_{G}}{C}=1 . \quad$ As $\frac{4 \pi d}{L}$ becomes large, $\frac{C_{G}}{C}=0.5$. For waves in very shallow water, the wave velocity is the same for all wave lengths and the group velocity is the same as the wave velocity. In deep water, the wave velocity depends on the wave length (dispersive medium) and the group velocity is one-half the wave velocity. The group velocity therefore falls in the range

$$
0.5 C<C_{G}<C
$$

Probably the most important application of the group velocity lies in the calculation of the rate of transmission of energy (18). Osborne Reynolds (19) showed for deep-water waves of sinusoidal form that one-half the energy was transmitted ahead. This result is in agreement with the conclusions of the trochoidal theory. However, for waves in any depth of water, Rayleigh (20) showed that the rate of transmission of energy is

$$
\frac{w C a^{2}}{4}\left(1+\frac{\frac{4 \pi d}{L}}{\sinh \frac{4 \pi d}{L}}\right)=\frac{w C a^{2}}{2} C_{G}
$$

Now $\frac{w C a^{2}}{2}$ is the entire wave energy which advances with the group velocity. This result is said to be characteristic of all waves and presumably could be applied to wave forms and velocity-length relationships found experimentally even though these results disagree with theory.

The general statement then appears to be that the power transmitted per unit of crest width is

$$
\begin{gathered}
P=\frac{C_{G} E}{L} \\
C_{G}=C-L \frac{d C}{d L}
\end{gathered}
$$

If waves in nature are found to be very nearly trochoidal, Gaillard's expression for the energy of both deep-water and shallow-water waves may be used for $E$ in equation 43. However, if experiment shows the trochoidal equations to be not sufficiently accurate, the true value is to be used in this equation, for the equation is general and does not depend upon any particular wave theory.

If experiment does not confirm equation 2 for the wave velocity as a function of wave length, the true experimental relationship is to be used in equation 44 for the group velocity. The derivative $\frac{d C}{d L}$ 
34

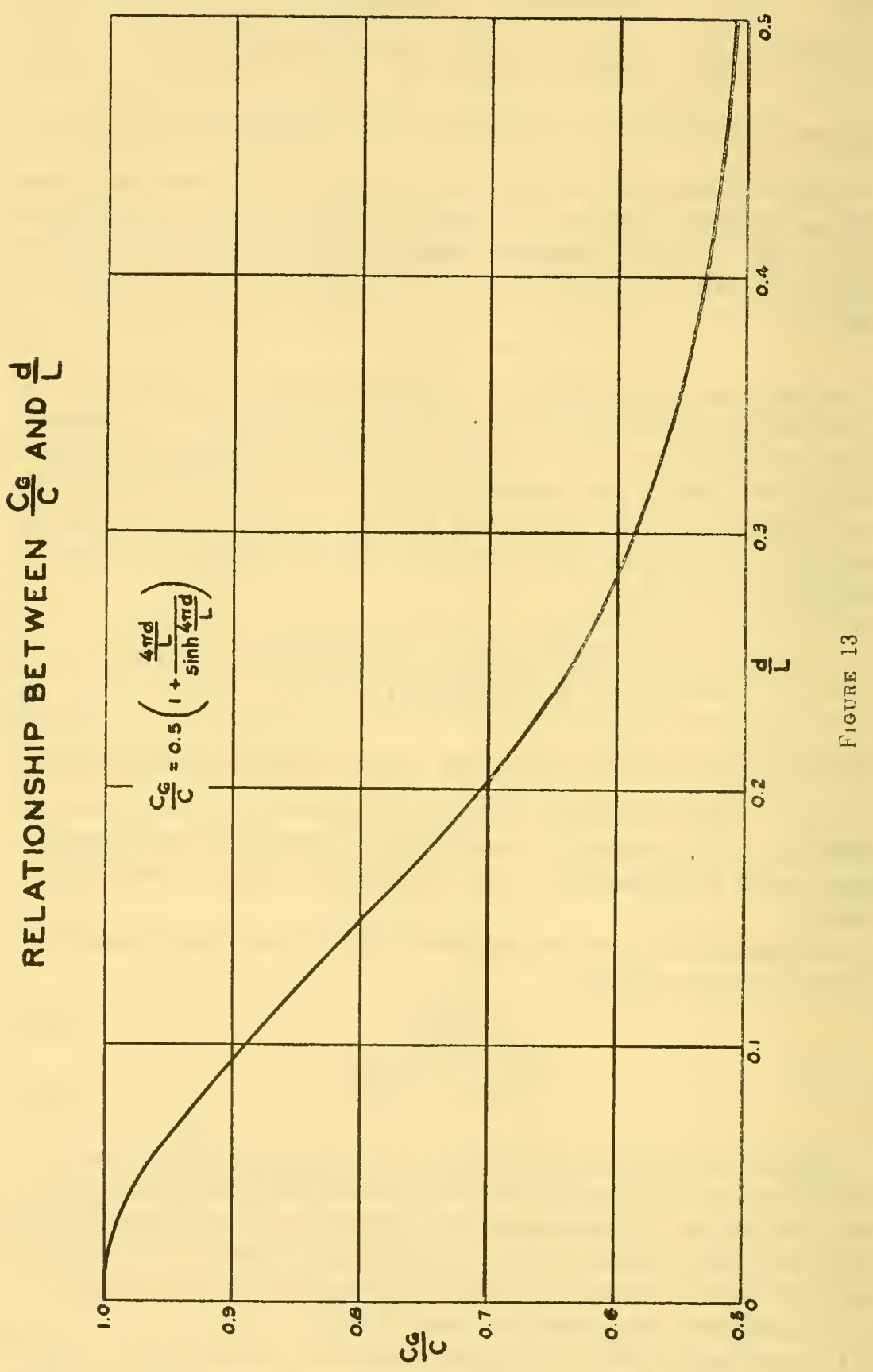


in equation 44 is to be obtained by differentiation with depth constant. If these data are represented graphically with $C$ a function of $L$ for different constant depths as parameter, tangents drawn to these curves will intercept the axis of $C(21)$ at the group velocity.

\section{Section 6. WAVES ON A SLOPING BOTTOM}

None of the theories previously discussed applies to waves moving over a sloping bottom. W. Wien (22) has considered the passage of a deep-water wave over a discontinuity in the bottom. Rayleigh (23) has discussed the variation in wave height on the assumption that waves in each depth on a sloping bottom have the same characteristics as in water of the same constant depth.

The changes in height and length which waves experience on running into shallow water is a well-known phenomenon. Quantitative knowledge of this effect is important in field studies. The depth in which the observations are made must be known in order to compute back to the characteristics of the same waves in deep water. The same problem arises in wave tank work where the most convenient method of characterizing the waves used in an experiment is by means of the computed characteristics in deep water.

Airy (24) makes an interesting statement regarding oscillatory waves over a shoaling bottom.

It would appear that when the depth is variable, it is impossible that there can be a series of waves which consist of oscillatory motion of the particles, and which satisfy the equations of continuity and equal pressure. The following physical interpretation of the mathematical result appears to be correct, and is worthy of attention. It appears that, if the water is moving in the manner of waves, one at least of the two conditions (continuity and equal pressure) must fail. While the continuity holds, the equal pressure will exist from the nature of the fluid. Therefore, the continuity must cease, or the water must become broken. This appears to be the explanation of the broken water which is usually seen upon the edge of a shoal or a ledge of rocks, although the whole is covered, perhaps deeply, by the water.

While Airy's conclusions appear to be substantiated by observation of waves moving over a deeply submerged reef, waves traveling on a gradually shoaling bottom do appear to move without breaking or losing their identity.

Stokes (2) commented on this problem as follows:

When swells are propagated toward a smooth, very gently shelving shore, the height increases where the finiteness of depth begins to take effect. * * * The breaking is no doubt influenced by friction against the bottom, but I do not believe that it is wholly or even mainly due to this cause. Before the wave breaks altogether the top gets very thin, but the maximum height for uniform propagation is probably already passed by a good deal, so that we must guard against being misled by this observation as to the character of the limiting form.

A surprising conclusion reached by Ivanov (25) is that the wave period depends upon water depth and bottom slope. For example, 
he found that at a slope $\frac{d}{L}=0.4$, the period increases 25 percent over the period in deep water. This conclusion is in confiict with wave tank observations and with one principal assumption running through these notes, namely, that the period of a single wave train is constant over a gradually sloping bottom. The problem should be given careful study experimentally.

Rayleigh (13) states that:

A uniform regime being established, what we are to equate at two separated places where the waves are of different character is the rate of propagation of energy through these places.

From his analysis he concludes that between very great depths and $\frac{d}{L}=0.04$ the bottom has little effect on the wave height but that:

* * * the wave length is diminishing, so that the waves, even though they do no more than maintain their height, grow steeper.

Although precise observations are necessary to establish the fact conclusively, it will be assumed as the most reasonable approach in the light of present knowledge that waves do maintain their identity in running over a gradually sloping bottom. Rayleigh's assumption will be made, namely, that the transmitted power is the same at all points along the path of travel.

The problem then is to trace a wave from deep water to the plunge point and predict the height, length, and velocity at each point along its path. The known characteristics in deep water are assumed to be the period and height although any other definitive combination is sufficient. From the period one obtains the velocity and length, which combined with the height gives the energy per wave length per unit width, and the transmitted power per unit width per second in deep water is found as:

$$
\begin{aligned}
& \mathrm{C}_{o}=5.12 T \\
& L_{o}=5.12 T^{2} \\
& P_{o}=\frac{w \sqrt{L} h^{2}}{7.05}\left(1-4.93 \frac{h^{2}}{L^{2}}\right)
\end{aligned}
$$

Consider a wave of uniform height with crest straight and parallel to the bottom contours. The problem is then two-dimensional. Furthermore, the effect of the finite height on the wave velocity will be neglected. In each depth, there is only one wave length and velocity consistent with the period. These values are obtained from figures 1 and 2 . The power is known from the computed value in deep water, equation 34 . 
Using the trochoidal theory formula for $E$, the height is obtained from equation 43 , since:

$$
E=\frac{w L h^{2}}{8}\left(1-M \frac{h^{2}}{L^{2}}\right)=\frac{P_{0} L}{C_{G}}
$$

Neglecting the quantity in parentheses, the approximate height is

$$
h^{\prime}=\frac{8 P_{o}}{\sqrt{w C_{G}}}
$$

Using this value of $h^{\prime}$ in the parentheses,

$$
h=\sqrt{\frac{8 P_{o}}{w C_{G}-\frac{S P_{o} M}{\left(L^{2}-M h^{2}\right)}}}
$$

The period $T$ is assumed to be constant, $P_{0}$ is known from the period and height in deep water and $L, M$, and $C_{G}$ are fixed by the period and depth, figures 2,12 , and 13 .

Since $\frac{C_{G}}{C}$ approaches unity as the depth decreases, the value of $h$

obtained from equation 46 will differ from that obtained from equation 38, which is based on the assumption that one-half of the energy travels with the wave form. Comparison of the heights predicted by equations 38 and 46 with experimental results should be decisive as to which concept of the energy transmission is correct.

If it is found that the wave velocity is appreciably affected by wave height, the quantities already obtained can be corrected, starting from the relationship $L=C_{h} T$. The corrected value of $L$ is then inserted in equation 46 in solving for $h$. It appears unlikely that the effect of finite height on velocity need be considered in engineering computations.

The computations cannot be carried as far as the plunge point. Independent experiments on the depth in which waves break must be relied upon for this limit. Insofar as the theory is concerned, the only limitation supplied is that the angle at the crest should not be less than $120^{\circ}$.

Dissipation of energy by internal and bottom friction before the wave reaches the outer line of breakers is assumed to be negligible. No assumption regarding reflection of the waves has been made but an experimental check on the theory would require that the energy be completely absorbed in order to permit accurate measurement of height and length.

\section{Section 7. WAVE REFRACTION}

The refraction of waves running obliquely on a shore line is a wellknown phenomenon. At the line of breakers, the wave crests seldom make an angle of more than $15^{\circ}$ with the shore line even when the crests in deep water are almost perpendicular to the shore. This 
bending of the waves so that the crests tend to conform to the bottom contours results from the reduction of wave velocity as the depth decreases; the inshore end of the wave travels at a lower velocity than the portion in deeper water. The phenomenon is known as wave refraction because of its similarity to the refraction of light and sound.

Consider the problem of the refraction of a wave approaching diagonally onto a straight shore line (all bottom contours parallel to shore). It is evident that each section of wave crest passes through the same sequence of angles and velocities and that the time history of the waves passing all points at equal distances from the shore will be the same except for the phase lag. The problem of determining the angle of the crest with the shore line at any point may be analyzed by a method suggested by C. K. Bagby.

Consider points $A$ and $B$, figure 14, separated by an infinitesimal distance $d s$. The velocity at $B$ exceeds that at $A$ and the wave front swings around as it advances during a short time interval $d t$. The change in angle of the wave front is given by

$$
\tan (-d \alpha)=-d \alpha=\frac{d C d t}{d s}=\frac{d C}{d y} \frac{d y}{d s} d t
$$

but: $\quad \frac{d y}{d s}=\sin \alpha$ and $\frac{d y}{d t}=-C \cos \alpha$, and the

differential equation may be expressed as

$$
\begin{aligned}
& \cot \alpha d \alpha=\frac{d C}{C} \\
& \log \sin \alpha=\log C+\text { constant }
\end{aligned}
$$

The boundary conditions are expressed in terms of the direction, $\alpha_{0}$, and velocity, $C_{o}$, in deep water as:

$$
\begin{aligned}
\text { constant } & =\log \sin \alpha_{o}-\log C_{o} \\
\sin \alpha & =\sin \alpha_{o} \frac{C}{C_{o}} \\
\alpha & =\sin ^{-1}\left(\frac{C}{C_{o}} \sin \alpha_{o}\right)
\end{aligned}
$$

It is noteworthy that the change in angle is not dependent upon the slope of the bottom, provided that the bottom contours are parallel to the shore and the slope gradual.

An auxiliary diagram will be convenient in determining the velocity as a function of depth and period (sec fig. 3).

The crest length will increase as a result of the refraction, the increase being in the ratio

$$
\frac{1}{\cos \left(\alpha_{o}-\alpha\right)}
$$


The more usual problem of wave refraction is that of a wave advancing to a curving shore line over a mildly irregular bottom. The methods outlined probably are inapplicable to abrupt changes in depth. The limitation, which can be stated only in very general terms until experimental results are available, is that the change in depth should be a small percentage of the depth in a distance equal to one wave length.

The problem requires graphical solution and the method employed is very nearly the same as for light and sound waves in which each point on the wave front is considered as a radiating center.

The time increment is usually taken as an integral multiple of the wave period. The accuracy of the diagram is increased by decreasing

\section{REFRACTION OF WAVES APPROACHING A SHORE}

SHORE

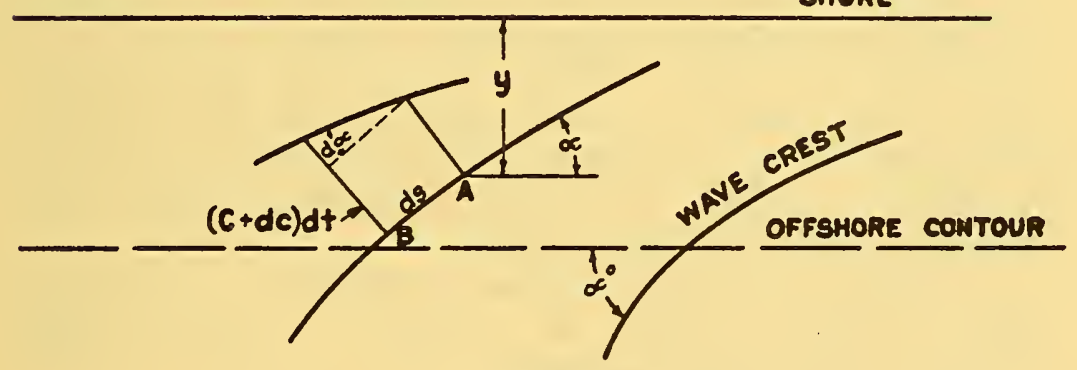

Figure 14.

the time interval. The criterion in choosing the time interval is that the difference in depth at the initial and final wave positions is a small percentage of the depth. It is seldom necessary to consider variations in tidal elevation in constructing these diagrams and the effect of finite wave height on wave velocity is neglected.

The wave height is obtained from a consideration of the power transmitted. If the wave crest in deep water is broken up into equal increments of length, lines drawn from the points of division and orthogonal to all of the wave crests will represent surfaces across which no power flows. The initial power is computed from the period and height by means of equations $2 c$ and 34 . On the assumption that frictional dissipation is negligible, the power per unit of crest 
length at a point at which the orthogonal lines are spaced at distance $b$ is

$$
\begin{aligned}
& P=\frac{b_{o}}{b} P_{o} \\
& h=\frac{8 b_{o} P_{o} T}{w b L C_{G}\left(1-M \frac{h^{2}}{L^{2}}\right)}
\end{aligned}
$$

All quantities in this equation are known except $h$ and the solution is made by approximation, assuming first that the quantity in parentheses (equation 45) is unity.

The steps in construction of wave refraction diagrams for a particular harbor or beach are:

1. Field data on periods, directions, and heights are first analyzed for frequency to discover the most frequent and important combinations of direction and period to be analyzed for refraction. For each important direction, one or more periods will be used.

2. For the chosen period, construct a diagram of wave velocity and length as a function of depth (figs. 1 and 2).

3. From inspection of the hydrographic chart choose a time interval such that, over most of the area, the wave advance during the interval will include a change in depth not over 10 percent of the depth. The time interval is best chosen as an integral multiple of the wave period.

4. Construct an auxiliary diagram showing the wave advance during the chosen interval as a function of depth. (Wave velocity times the chosen interval.)

5. Place a transparent overlay on the hydrographic chart and insert the initial wave crest position in deep water. The wave will advance parallel to itself by equal increments until the depth begins to affect the velocity. Usually, the effect of the bottom is not appreciable until the depth has been reduced to about 30 percent of the deep-water length.

6 . Using the graph constructed under 4 lay off distances perpendicular to the wave front corresponding to each depth along the crest. A refinement in the method consists in finding the approximate new depth and then laying off a perpendicular distance corresponding to the mean depth in the interval.

7. Strictly speaking the wave advances in 6 should be drawn as arcs and a new wave crest drawn as the envelope of these arcs. If the time interval is not too long, the pependicular distance will be correct within the usual accuracy of such diagrams.

8. Continue this procedure to the point at which the smallest wave of interest in the study would break. 
9. Divide the initial wave crest in deep water into equal increments and draw lines perpendicular to all intermediate wave crests from these division points to the shore.

10. From the known period and height in deep water, compute the power per unit of crest length and the height of the same wave at different points in the diagram. The power per unit of crest length is inversely proportional to the spacing of the orthogonal lines measured along the wave crest.

The method outlined is applicable to a gradually sloping bottom. Very steep or vertical slopes, as around the end of a breakwater built ir deep water, present problems, especially in estimating the heights, which can only be solved by supplementing this method with experimental results.

\section{Section 8. DAMPING OF OSCILLATORY WAVES}

After the forces generating waves cease to operate, the waves run freely, losing energy by friction as they proceed. Waves in nature probably are reduced in height by an increase in wave length as they move outward from the source. In deep water, internal friction converts mechanical energy into heat, while in shallow water both internal and bottom friction are operative. Waves generated in tanks also lose energy by friction on the walls. The problem has been analyzed theoretically by a number of investigators. Only a brief summary of the general results will be presented here. Damping of waves in tanks by bottom and wall friction is to be considered in a separate report.

Wave motion requires a distortion of the fluid elements and therefore viscous damping will occur even in deep water and in the absence of vertical walls. The problem has been treated by Lamb (26) as follows:

If the wave surface profile is represented by the equation

$$
y=\frac{h}{2} \sin \frac{2 \pi}{L}(x-C t)
$$

where $h=$ full amplitude (trough to crest)

the average kinetic energy per unit area of surface is $1 / 16 \rho m h^{2} C^{2}$ and the total energy is twice that, or $1 / 8 \rho m h^{2} C^{2}$. From analysis of the rate of distortion of fluid elements, the average rate of dissipation per unit area is found to be

$$
-\frac{1}{2} \mu m^{3} C^{2} h^{2}
$$


Therefore

$$
\begin{aligned}
\frac{d}{d t}\left(\frac{1}{2} \rho m C^{2} h^{2}\right) & =-\frac{1}{2} \mu m^{3} C^{2} h^{2} \\
\frac{d h}{d t} & =-2 \nu h m^{2} \\
h & =h_{o} e^{-2 \nu t m^{2}}
\end{aligned}
$$

The time, $t_{r}$ necessary for the height to be reduced in the ratio $e: 1$ is

$$
t_{r}=\frac{1}{2 \nu m^{2}}=\frac{L^{2}}{8 \nu \pi^{2}}
$$

Assuming that the wave length-velocity-period relationship is unaffected by viscosity

$$
L=5.12 T^{2} \quad C=5.12 T
$$

and the distance $L_{\tau}$ of wave travel corresponding to the same ratio of

\begin{tabular}{|c|c|c|c|}
\hline Wave period (seconds) & $\begin{array}{c}\text { Wave length } \\
\text { (feet) }\end{array}$ & $\begin{array}{l}\text { Wave velocity } \\
\text { (feet/second) }\end{array}$ & $\begin{array}{l}\text { Wave travel } \\
\text { (miles) }\end{array}$ \\
\hline $1{ }^{1}$ & $\begin{array}{l}5.1 \\
128 \\
512\end{array}$ & $\begin{array}{r}5.1 \\
25.6 \\
51.2\end{array}$ & $\begin{array}{l}32.5 \\
102,000 \\
3,250,000\end{array}$ \\
\hline
\end{tabular}
height reduction is

$$
\begin{gathered}
L_{r}=C t_{r}=1.71 \frac{T^{5}}{\nu} \\
\nu=1.0 \times 10^{-5} f t^{2} / \mathrm{sec} . \quad L_{r}=1.71 \times 10^{5} T^{6}
\end{gathered}
$$

Wave travel required for a ratio of reduction in height of $e: 1$

The theory shows a tremendously slow rate of damping by internal friction for even short period waves.

The theory outlined starts from the assumption of a sinusoidal wave form but the theory may be taken as indicating a very slow rate of damping of all waves in deep water.

The damping of waves in shallow water has been considered by Hough (27) who also found that the modulus of decay, or time of travel necessary for the wave to be reduced in amplitude in the ratio $e: 1$ is

$$
t_{\tau}=\frac{L^{2}}{8 \nu \pi^{2}}
$$

For fluids having small viscosities, such as water, the velocity of the wave and the relationship between depth, length, and period is unaltered by friction. Hough states that:

This agrees with the formula given by Professor Lamb for the case of waves in deep water. We see now that it holds for waves of any wave-length in water of any depth, provided that the bottom is perfectly smooth and that the internal viscosity is sufficiently small to allow of our approximations. 
In nature, the dissipation of energy is very gradual and correction for energy dissipation may be unnecessary. If a correction must be made in wave refraction studies, the computation proceeds by a series of approximations using the heights computed on the assumption of no energy loss as a first approximation.

Equation 54, which applies to all waves in all depths of water, was derived on the assumption that the bottom is perfectly smooth and the flow viscous. Remembering that the frictionless case gives large velocities at the bottom in shallow water and that the bottom in nature is always rough, it appears likely that turbulent friction at the bottom is present in the usual case. Compared with the probable thickness of the boundary layer, even sand grains in a smooth layer present a considerable roughness. The bottom is generally marked by ripples and observation shows that vortices do form around these ripples. In very shallow water, there are often observed clouds of sand which may result from hydraulic flow in the sand from points under the crest to the trough, but which certainly indicate that the motion near the bottom is not laminar whatever may be the origin of the clouds. 




